\title{
Theoretical and experimental comparative analysis of beamforming methods for loudspeaker arrays under given performance constraints
}

\author{
Ferdinando Olivieri, Filippo Maria Fazi, Philip A. Nelson, Mincheol Shin \\ Institute of Sound and Vibration Research, University of Southampton, Southampton, SO17 1BJ, UK. \\ Simone Fontana, Lang Yue \\ Huawei European Research Center, Riesstraße 25, 80992, Munich, Germany.
}

\begin{abstract}
Methods for beamforming are available that provide the signals used to drive an array of sources for the implementation of systems for the so-called personal audio. In this work, performance of the delay-and-sum (DAS) method and of three widely used methods for optimal beamforming are compared by means of computer simulations and experiments in an anechoic environment using a linear array of sources with given constraints on quality of the reproduced field at the listener's position and limit to input energy to the array. Using the DAS method as a benchmark for performance, the frequency domain responses of the loudspeaker filters can be characterized in three regions. In the first region, at low frequencies, input signals designed with the optimal methods are identical and provide higher directivity performance than that of the DAS. In the second region, performance of the optimal methods are similar to the DAS method. The third region starts above the limit due to spatial aliasing. A method is presented to estimate the boundaries of these regions.
\end{abstract}

Preprint submitted to Journal of Sound and Vibration

February 7, 2016 


\section{Contents}

1 Introduction 44

2 Theoretical preliminaries $\quad 6$

3 Signal processing for beamforming with loudspeaker arrays $\quad 8$

3.1 Delay-and-sum beamformer with near-field amplitude compensation . . . . . . . 8

3.2 Pressure Matching $(\mathrm{PM}) \operatorname{method} \ldots \ldots \ldots \ldots$

3.3 Acoustic Contrast Maximization $(\mathrm{ACM}) \ldots \ldots \ldots \ldots$

3.4 Energy Difference Maximization $(\mathrm{EDM}) \ldots \ldots \ldots \ldots \ldots$

4 Strategy for comparison of beamforming methods 11

4.1 Theoretical considerations on the compensated filters . . . . . . . . . . . . 14

4.2 Implementation of the performance constraints . . . . . . . . . . . . . . . . 14

5 Validation of the proposed strategy for comparison of beamforming methods 16

5.1 Linear array prototype and definition of the beamforming problem . . . . . . . 16

5.2 Design of the compensated filters . . . . . . . . . . . . . 16

5.3 Simulations and Experiments . . . . . . . . . . . . . . . . 18

$5.3 .1 \quad$ Experimental setup . . . . . . . . . . . . . . . . . 18

5.3.2 Electro-acoustical analysis of the array . . . . . . . . . . . . . . 19

5.3.3 Performance parameters . . . . . . . . . . . . 20

5.4 Results for the front beam . . . . . . . . . . . . . . . . 23

5.5 Discussion of results $\ldots \ldots \ldots \ldots \ldots \ldots$

5.6 Results for the side beam . . . . . . . . . . . . . 36

6 Conclusions $\quad 41$

Appendix A Proof of PM compensated solution 42

Appendix B Procedure for the calculation of $\alpha_{\mathrm{MAX}}$ 


\section{Highlights}

6 Note to the editor

$7 \quad$ Part of this work was presented in [1].

\section{Classification}

$9 \quad$ K Signal processing for sound and vibration applications 


\section{Introduction}

Purposefully designed input signals can be used to control the directivity pattern of an array of loudspeakers of compact form factor for the implementation of personal audio systems [2, 3].

This is usually achieved by focusing sound in a given region of the space, hereafter also referred to as acoustically bright area, while minimizing sound radiation elsewhere. The acoustically bright area may consist of a number of so-called control points [3] or a single control point [25]. In the first case, we refer to it as the process of generating private sound zones, whilst in the second case, which is the one considered in this work, we aim at the generation of private sound beams, hereafter also succinctly referred to as sound beams.

Input signals that allow for the creation of private sound beams can be designed using methods for beamforming for loudspeaker arrays, such as the delay-and-sum (DAS) method or through the application of Time-Reversal signal processing [4], to cite a few. From 1990 onwards, research has focused on increasing directivity performance of loudspeaker arrays by means of optimal beamforming methods which can be broadly divided into three categories. 1) Methods that aim at the reproduction (or synthesis) of a target field, such as the Pressure-Matching (PM) method [5]. 2) Methods for the control of the acoustic energy density in the control zone, hereafter also referred to as energy based methods, such as the Acoustic Contrast Maximization $[3,6]$ (ACM), and the Energy Difference Maximization [7, 8] (EDM) methods. 3) Hybrid methods that combine the synthesis of a target field and the control of the acoustic energy density $[9,10,11]$. In general, for a given configuration of loudspeaker array and control area, input signals designed with a given method for beamforming may depend on a number of tunable parameters, such as the regularization parameters for the PM and ACM methods. Due to these differences, a comparison between strategies for beamforming is not straightforward. Methods for beamforming for loudspeaker arrays have been previously compared for various geometrical arrangements and applications, such as in personalized audio systems for vehicles [12], generation of warning signals for the safety of pedestrians [13], and for loudspeaker arrays operating in a reflective environments [14] or under free-field conditions [3,15, 16, 17]. Trade-offs between concurring performance factors, such as directivity performance $[3,15,17,28]$, input energy required to control sound field $[3,17,28]$, robustness of the system against mismatch of the responses between sources and control points $[3,17]$, and frequency response of the reproduced field $[12,15]$ have 
been reported in the literature as well as the fact that system performance is highly dependent on the choice of tunable parameters of a given beamforming method [8, 15, 16, 17].

The increasing demand for high-performance portable devices for communications, such as tablet or mobile phones, suggests that systems for personalized audio should be able to provide listeners with a suitable sound pressure level over a broad frequency range (hereafter succinctly referred to as audio quality) and limited energy consumption. These two concurring performance constraints form the basis of the comparison between beamforming methods proposed in this work. More specifically, input signals generated under the above-mentioned performance constraints with four widely used beamforming methods for loudspeaker arrays, i.e. the delay-and-sum (DAS) beamformer, the PM, the ACM, and the EDM methods, are compared using a linear 11 array prototype by means of numerical simulations and experiments in an anechoic environment. 12 A strategy is presented to calculate the parameters of each method (such as the regularization 13 parameter) in order to ensure that input signals designed with the methods considered satisfy the same constraints in terms of audio quality and required input energy at each frequency. The main result of this work is that, based on the given constraints, performance of the optimal methods considered relate to each other and to that of the DAS as a function of frequency. More specifically, they are identical at low frequencies and they resemble that of the DAS in the mid-frequency range. A method is presented to estimate the frequencies at which the boundaries of these regions occur. Moreover, the results of the experiments with the real loudspeaker array show the effects of the mismatches between transducers of the array on system robustness, the latter being discussed and analyzed by using the measured transfer functions of the array in the anechoic chamber. The remainder of the paper is organized as follows. The theoretical preliminaries are introduced in Section 2. A review of the beamforming methods considered in this work, their theoretical model and their differences are reported in Section 3. The strategy for the beamforming method comparison is described in Section 4. The details of the linear array, the analysis of the designed filters, the numerical and experimental validations are described in Section 5 . The conclusions are drawn in Section 6. 


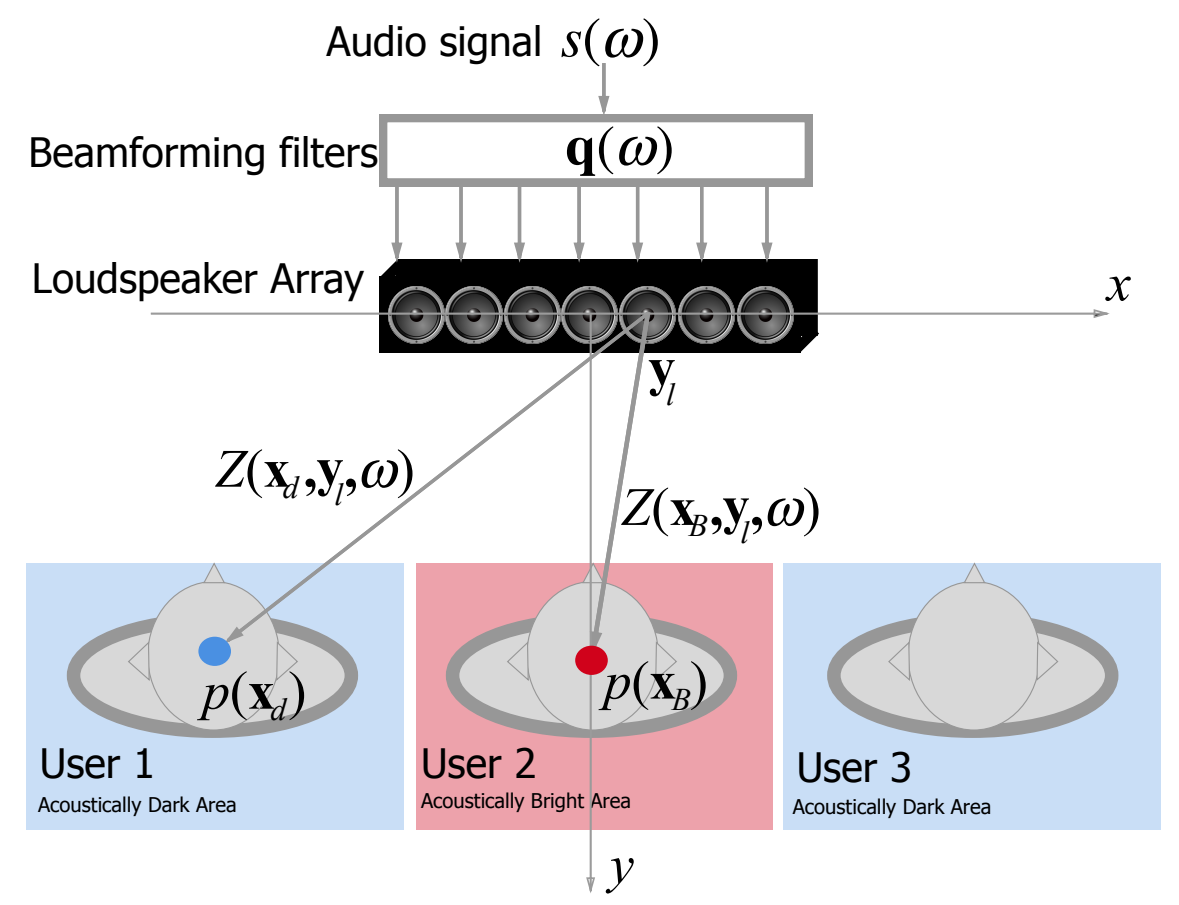

Figure 1: (Color online) Example of a beamforming problem for personalized audio applications. A loudspeaker array is driven by purposefully designed input signals to focus sound where User 2 is located while minimizing sound pressure where User 1 and User 3 are located.

\section{Theoretical preliminaries}

The beamforming problem in this work is formulated for a given angular frequency $\omega=2 \pi f$,

3 where $f$ is the temporal frequency, and all the acoustical quantities introduced in this section have

4 a time dependence of $e^{j \omega t}$, where $j=\sqrt{-1}$ is the imaginary unit and $t$ is the time. With reference

5 to Fig. 1, let $s(\omega)$ be the spectrum of the mono signal that we want to deliver to the listener. Let us

6 also consider an array of $L$ sources of coordinates $\mathbf{y}_{\ell}, \ell \in[-(L-1) / 2,(L-1) / 2]$, which are driven

7 by the input signals $s(\omega) \mathbf{q}(\omega)$, where $\mathbf{q}(\omega) \in \mathbb{C}^{L \times 1}, \mathbf{q}^{T}(\omega)=\left[q_{1}(\omega), \ldots, q_{L}(\omega)\right]$, is the vector

8 of the frequency response functions (FRF) at frequency $\omega$ of the beamforming filters designed

$9 \quad$ with a given method for beamforming and $[\cdot]^{T}$ is the operation of vector (of matrix) transpose.

10 The control area is typically sampled over a finite number $M$ of control points of coordinates $\mathbf{x}_{m}$,

$11 m=1, \cdots, M$. Virtual microphones are placed at the locations of the control points to estimate

12 the sound pressures due to the array of sources driven with $s(\omega) \mathbf{q}(\omega)$. The output signals from 
1 the virtual microphones are indicated by $\mathbf{p}(\omega) \in \mathbb{C}^{M \times 1}, \mathbf{p}^{T}=\left[p\left(\mathbf{x}_{1}, \omega\right), \ldots, p\left(\mathbf{x}_{M}, \omega\right)\right]$. The 2 assumption is made that the relation between $\mathbf{p}(\omega)$ and $s(\omega) \mathbf{q}(\omega)$ is linear, that is [5]

$$
\mathbf{p}(\omega)=\mathbf{Z}(\omega) \mathbf{q}(\omega) s(\omega)
$$

where $\mathbf{Z}(\omega) \in \mathbb{C}^{M \times L}$ is the matrix of transfer functions (TFs) between the virtual microphone signals and the input signals. The sources of the array are assumed to radiate as ideal monopole sources in free-field and the $(m, \ell)$-th element $Z_{m, \ell}(\omega)$ of the TFs matrix is, therefore,

$$
Z_{m, \ell}(\omega)=Z\left(\mathbf{x}_{m}, \mathbf{y}_{\ell}, \omega\right)=\frac{e^{-j \frac{\omega}{c}\left\|\mathbf{x}_{m}-\mathbf{y}_{\ell}\right\|}}{4 \pi\left\|\mathbf{x}_{m}-\mathbf{y}_{\ell}\right\|},
$$

6 where $c$ is the speed of sound and $\|\cdot\|$ is the $l_{2}$-norm operator. Hereafter, in order to simplify 7 the notation, the dependence of the acoustical quantities on the angular frequency $\omega$ is omitted.

8 Depending on whether sound is desired or undesired at a given control point, the latter could 9 be either labeled as acoustically bright or acoustically dark (or, more succinctly, bright or dark 10 point), respectively. The vector of coordinates of the bright point (where the listener is located) is 11 indicated by $\mathbf{x}_{B}$. The microphone signal at the bright point $\mathbf{x}_{B}$ is denoted by $p_{B}=p\left(\mathbf{x}_{B}\right)=\mathbf{z}_{B}^{T} \mathbf{q}$, 12 where $\mathbf{z}_{B}^{T}=\left[Z\left(\mathbf{x}_{B}, \mathbf{y}_{1}\right), \cdots, Z\left(\mathbf{x}_{B}, \mathbf{y}_{L}\right)\right]$ is the vector of TFs between each source of the array and the bright point. The coordinate of the $d$-th control point in the dark zone, $d=1, \cdots, M_{D}$, where $M_{D}$ is the number of control points in the dark zone, is denoted by $\mathbf{x}_{d}^{(D)}$. The sets of $M_{B}=1$ bright point and $M_{D}$ dark points form the acoustically bright and acoustically dark zone, respectively, and $M_{B}+M_{D}=M$. Having introduced the notation for bright and dark points, the vector of microphone signals $\mathbf{p}$ and the TF matrix $\mathbf{Z}$ can also be expressed as

$$
\mathbf{p}=\left[\begin{array}{c}
p_{B} \\
\mathbf{p}_{D}
\end{array}\right], \quad \mathbf{Z}=\left[\begin{array}{c}
\mathbf{z}_{B}^{T} \\
\mathbf{z}_{D}
\end{array}\right],
$$

where $\mathbf{p}_{D}=\mathbf{Z}_{D} \mathbf{q}$ are the microphone signals defined at the control points in the dark zones. Matrix $\mathbf{Z}_{D}$ is the $M_{D} \times L$ TF matrix whose rows are the vectors of the transfer functions between the sources and the control points in the dark zone. 


\section{Signal processing for beamforming with loudspeaker arrays}

$2 \quad$ The beamforming filters $\mathbf{q}$ are designed by setting $s(\omega)=1$ on a frequency-by-frequency 3 basis for each frequency in the range of interest, which typically ranges from $-f_{s}$ to $f_{s}$, where

$4 f_{S}$ is the sampling frequency. This frequency range is sampled at a number $N_{\mathrm{FFT}}$ of frequency

5 bins with uniform frequency spacing $\Delta f$. The time-domain input signals to the array are then 6 obtained by inverse Fourier transform of the values of the elements of $\mathbf{q}$, provided that filters are 7 made causal by applying a so-called modeling delay $e^{-j \omega \Delta t}[18]$, where $\Delta t$ is the modeling delay 8 in seconds.

\section{3.1. Delay-and-sum beamformer with near-field amplitude compensation} are such to produce unitary pressure at the bright point and they are calculated as

$$
\mathbf{q}_{\mathrm{DAS}}=\Gamma \mathbf{z}_{B}^{*},
$$

$$
\gamma_{\ell}=\frac{\left(4 \pi r_{\ell}\right)^{2}}{L}
$$

where $[\cdot]^{*}$ is the operation of vector (or matrix) complex conjugate and $\boldsymbol{\Gamma}$ is a diagonal matrix of dimension $L \times L$ which is used for the near-field compensation and whose $\ell$-th element $\gamma_{\ell}$ on the main diagonal is

where $r_{\ell}=\left\|\mathbf{x}_{B}-\mathbf{y}_{\ell}\right\|$. Beside the DAS method, whose performance is used in this work as a benchmark, strategies for optimal beamforming, also referred to as super-directive beamforming, are available. Typically, filters designed with approaches for optimal beamforming are obtained by minimization/maximization of a given cost function.

\subsection{Pressure Matching (PM) method}

Let $\hat{\mathbf{p}}^{T}=\left[\hat{p}\left(\mathbf{x}_{1}\right), \ldots, \hat{p}\left(\mathbf{x}_{M}\right)\right]$ be a desired target field defined at the $M$ control points. The filters that allow for the synthesis of $\hat{\mathbf{p}}$ can be designed using the Pressure Matching (PM) method 
1 by minimizing the cost function $J_{\mathrm{PM}}=\mathbf{e}_{\mathrm{PM}}^{H} \mathbf{e}_{\mathrm{PM}}+\beta_{\mathrm{PM}} E_{\mathbf{q}}[5,19,20]$, where $[\cdot]^{H}$ is the operator 2 of complex conjugate transpose, that is

$$
\underset{\mathbf{q}}{\operatorname{minimize}} J_{\mathrm{PM}}=\underset{\mathbf{q}}{\operatorname{minimize}}\left(\mathbf{e}_{\mathrm{PM}}^{H} \mathbf{e}_{\mathrm{PM}}+\beta_{\mathrm{PM}} E_{\mathbf{q}}\right),
$$

3 where $\mathbf{e}_{\mathrm{PM}}=\hat{\mathbf{p}}-\mathbf{Z q}$ is the complex error between the target field and the reproduced pressure $4 \quad[5,20]$ and $E_{\mathbf{q}}$ is the input energy to the array of sources, also referred to as array effort, that is 5 defined as

$$
E_{\mathbf{q}}=\|\mathbf{q}\|^{2}=\mathbf{q}^{H} \mathbf{q}
$$

6 and $\beta_{\mathrm{PM}} \in[0, \infty)$ is the Tikhonov regularization parameter, which can be frequency dependent

$7 \quad[5,20]$. The array effort $E_{\mathbf{q}}$ is often used as a measure of the input energy required by the array to 8 control the sound field at a given frequency [3]. It can be shown that $J_{\mathrm{PM}}$ is convex with respect to 9 the real and imaginary parts of $\mathbf{q}$ and it has a unique global minimum $\mathbf{q}_{\mathrm{PM}}$ [19] (i.e., the optimal 10 filters in a least-squares sense). The vector $\mathbf{q}_{\mathrm{PM}}$ is found by setting to zero the complex gradient

11 of $J_{\mathrm{PM}}$ with respect to $\mathbf{q}$ and then solving with respect to $\mathbf{q}$ [20]. Assuming $M>L$, $\mathbf{q}_{\mathrm{PM}}$ is given

12 by $[19,20]$

$$
\mathbf{q}_{\mathrm{PM}}=\left(\mathbf{Z}^{H} \mathbf{Z}+\beta_{\mathrm{PM}} \mathbf{I}\right)^{-1} \mathbf{Z}^{H} \hat{\mathbf{p}}
$$

13 where $(\cdot)^{-1}$ indicates the operation of matrix inversion, and $\mathbf{I}$ is the identity matrix of dimensions $14 L \times L$. In personalized audio applications, the target field $\hat{\mathbf{p}}$ for the PM method is commonly 15 defined as $[15,20]$

$$
\hat{\mathbf{p}}=\left[\begin{array}{c}
\hat{p}_{B} \\
\hat{\mathbf{p}}_{D}
\end{array}\right],
$$

16 where $\hat{p}_{B}=1$ is the target signal at the bright point, $\hat{\mathbf{p}}_{D}^{T}=\mathbf{0}_{M_{D}}$ is the target signal in the dark zone,

17 and $\mathbf{0}_{M}=[0,0, \cdots, 0]^{T}$ is the vector of length $M$ whose elements are all equal to 0. 


\subsection{Acoustic Contrast Maximization (ACM)}

2 Let us define the acoustic potential energy density, hereafter referred to also as acoustic 3 energy, at the bright point as [6, 7]

$$
E_{B}=\frac{1}{\rho_{0} c^{2}}\left|p_{B}\right|^{2}=\frac{1}{\rho_{0} c^{2}} \mathbf{q}^{H} \mathbf{R}_{B} \mathbf{q}
$$

${ }_{4}$ where $\rho_{0}$ is the density of the medium and $\mathbf{R}_{B}=\mathbf{z}_{B}^{H} \mathbf{z}_{B}$ represents the spatial correlation of the 5 transfer functions vector $\mathbf{z}_{B}[6,8]$. Similarly, we define the acoustic energy $E_{D}$ in the dark zone $6 \quad$ spatially averaged over $M_{D}$ dark points as

$$
E_{D}=\frac{1}{\rho_{0} c^{2}} \frac{1}{M_{D}}\left\|\mathbf{p}_{D}\right\|^{2}=\frac{1}{\rho_{0} c^{2}} \mathbf{q}^{H} \mathbf{R}_{D} \mathbf{q}
$$

7 where $\mathbf{R}_{D}=\mathbf{Z}_{D}^{H} \mathbf{Z}_{D} / M_{D}$ represents the spatial correlation of the transfer functions matrix $\mathbf{Z}_{D}$ $8 \quad[6,8]$. The acoustic contrast $\mathrm{AC}$ is defined as the ratio between $E_{B}$ and $E_{D}$, that is [6]

$$
\mathrm{AC}=\frac{E_{B}}{E_{D}}=\frac{\mathbf{q}^{H} \mathbf{R}_{B} \mathbf{q}}{\mathbf{q}^{H} \mathbf{R}_{D} \mathbf{q}},
$$

and it is a dimensionless unit often used as a measure of directivity performance of the array.

The Acoustic Contrast Maximization (ACM) method, introduced by Choi et al. [6], aims at

11 finding the filters that maximize the cost function $J_{\mathrm{ACM}}=\mathrm{AC}$. The corresponding maximization

12 problem can be written as [6]

$$
\underset{\mathbf{q}}{\operatorname{maximize}} J_{\mathrm{ACM}}=\underset{\mathbf{q}}{\operatorname{maximize}} \frac{\mathbf{q}^{H} \mathbf{R}_{B} \mathbf{q}}{\mathbf{q}^{H} \mathbf{R}_{D} \mathbf{q}} .
$$

13 The vector $\mathbf{q}_{\mathrm{ACM}}$ that maximizes $J_{\mathrm{ACM}}$ is given by the following eigenvalue problem $[6,3]$

${ }_{14} \lambda_{n} \mathbf{q}_{n}=\left(\mathbf{R}_{D}^{-1} \mathbf{R}_{B}\right) \mathbf{q}_{n}$, where $\lambda_{n}$ is the $n$-th eigenvalue of matrix $\mathbf{R}_{D}^{-1} \mathbf{R}_{B}, n=1, \cdots, N$, and $N$ is

15 the number of eigenvalues of matrix $\mathbf{R}_{D}^{-1} \mathbf{R}_{B}$. The vector $\mathbf{q}_{\mathrm{ACM}}$ is calculated by selecting the

16 eigenvector corresponding to the maximum eigenvalue of the matrix $\mathbf{R}_{D}^{-1} \mathbf{R}_{B}$ [6], that is

$$
\mathbf{q}_{\mathrm{ACM}}=\Phi\left(\mathbf{R}_{D}^{-1} \mathbf{R}_{B}\right)
$$

17

where $\Phi(\mathbf{A})$ is a the operator that returns the eigenvector corresponding to the maximum eigenvalue of a matrix A. Due to the presence of the matrix inversion, a regularization parameter is 
1 needed to reduce the effects of the ill-conditioning of matrix $\mathbf{R}_{D}$. Hence, $\mathbf{q}_{\mathrm{ACM}}$ is calculated from $2 \quad[6]$

$$
\mathbf{q}_{\mathrm{ACM}}=\Phi\left(\left(\mathbf{R}_{D}+\beta_{\mathrm{ACM}} \mathbf{I}\right)^{-1} \mathbf{R}_{B}\right)
$$

where $\beta_{\mathrm{ACM}} \in[0, \infty]$ is the regularization parameter.

4.4. Energy Difference Maximization (EDM)

Shin et al. [7] proposed an energy-based method that aims at finding the filters $\mathbf{q}$ that 6 maximize the difference between the acoustic energy density in the bright zone $E_{B}$ and the 7 acoustic energy density in the dark zone $E_{D}$, the latter weighted by a positive real-valued factor $\alpha$

8 [7]. Due to this reason, the method is named Energy Difference Maximization (EDM) and the 9 corresponding maximization problem is defined as [7]

$$
\underset{\mathbf{q}}{\operatorname{maximize}} \frac{E_{B}-\alpha E_{D}}{E_{\mathbf{q}}}=\underset{\mathbf{q}}{\operatorname{maximize}} \frac{\mathbf{q}^{H}\left(\mathbf{R}_{B}-\alpha \mathbf{R}_{D}\right) \mathbf{q}}{\mathbf{q}^{H} \mathbf{q}} .
$$
to the maximum eigenvalue of the matrix $\mathbf{R}_{B}-\alpha \mathbf{R}_{D}$, that is

$$
\mathbf{q}_{\mathrm{EDM}}=\Phi\left(\mathbf{R}_{B}-\alpha \mathbf{R}_{D}\right)
$$

13 Most noticeably, the EDM method does not involve the operation of matrix inversion.

\section{4. Strategy for comparison of beamforming methods}

\footnotetext{
There are a number of differences between the beamforming methods considered in this work. For example, the PM method allows for control of the reproduced field at the control points in magnitude and phase (through the synthesis of a desired field). On the other hand, energy-based methods (like the ACM and EDM) aim at controlling the acoustic energy density in the control zone, thus a quantity that is proportional to the magnitude squared of the reproduced field. For this reason, energy-based methods are referred to as being phase ambiguous [17].

This, in turn, may potentially lead to audio quality issues at the listener's position [8]. In
} 
1 order to overcome this problem, Shin et al. [8] have proposed a modified version $\tilde{\mathbf{q}}_{\mathrm{EDM}}$ of the filters $\mathbf{q E D M}_{\mathrm{EDM}}$ calculated with the EDM method, that is $\tilde{\mathbf{q}}_{\mathrm{EDM}}=\hat{W}_{\mathrm{EDM}} \mathbf{q}_{\mathrm{EDM}}$, where $\hat{W}_{\mathrm{EDM}} \in \mathbb{C}$, $\hat{W}_{\mathrm{EDM}}=\left(\hat{p}_{B} /\left|\hat{p}_{B}\right|\right) \cdot\left(\left|\mathbf{z}_{B}^{T} \mathbf{q}_{\mathrm{EDM}}\right| / \mathbf{z}_{B}^{T} \mathbf{q}_{\mathrm{EDM}}\right)$, is an all-pass filter for the compensation of the phase response of the reproduced field with respect to the response of a desired signal $\hat{p}_{B}$, both defined at the bright point [8].

In addition to the differences in types of sound field control, for given configurations of array and control point geometries, beamforming methods may require the setting of the values of the so-called tunable parameters, such as the regularization parameters $\beta_{\mathrm{PM}}$ and $\beta_{\mathrm{ACM}}$ in the $\mathrm{PM}$ and ACM methods, respectively, and $\alpha$ in the EDM method. Those parameters have, in general, a significant impact on the performance of the system for personalized audio [8]. For example, the regularization parameter $\beta_{\mathrm{PM}}$ in the PM method controls the trade-off between the directivity performance and the input energy required by the array. Instead, the regularization parameter $\beta_{\mathrm{ACM}}$ in the formulation of the ACM method given in Eq. (15) does not control the input energy to the array. This is due to the fact that the filters for the ACM method (as well as the EDM method) are calculated by selecting an eigenvector of a matrix and the $l_{2}$-norm squared of this eigenvector (i.e., the input energy) is unitary by definition.

Comparisons of optimal methods have been proposed in the literature. Betlehem et al. [28] reported that PM and ACM solutions are identical if the choice of the target field in the bright zone is an ACM solution and if the designed input signals (with PM and ACM) meet identical constraints on the input energy and level of the energy of the reproduced field in the dark zone. This concept was proven experimentally by Simón Gálvez et al. [15]. Similarly, Elliott et al. [3] compared the regularized formulations of energy-based methods (e.g., ACM and EDM) and discussed their similarity depending on the choice of the values of the regularization parameters to meet given performance constraints.

As previously stated, in order to enable a fair comparison between inputs signals designed with the considered beamforming methods, two constraints on performance are introduced. The first performance constraint is set to ensure that the designed filters provide accurate reproduction of a target signal $\hat{p}_{B}$. In order to meet the first constraint, filters $\mathbf{q}$ designed with a given beamforming method are multiplied by a filter $W \in \mathbb{C}$ for compensation of the magnitude and phase of the reproduced pressure at the bright point, thus giving the compensated filters $\hat{\mathbf{q}}^{T}=\left[\hat{q}_{1}, \ldots, \hat{q}_{L}\right]$, that 
1 are defined as

$$
\hat{\mathbf{q}}=W \mathbf{q},
$$

2 where $W$ is the compensation filter defined as

$$
W=\frac{\hat{p}_{B}}{\mathbf{z}_{B}^{T} \mathbf{q}},
$$

3 which extends the "phase-compensation" filter used in [8]. The first constraint can be written as

$$
p_{B}(\hat{\mathbf{q}})=\hat{p}_{B}
$$

4 and this accounts for the quality of the pressure field at the listener's position.

5 Due to the fact that loudspeaker arrays can handle limited input energy, a second performance 6 constraint is set to ensure that the energy $E_{\hat{\mathbf{q}}}=\|\hat{\mathbf{q}}\|^{2}$ of the compensated filters $\hat{\mathbf{q}}$ does not exceed 7 a given maximum value $E_{\mathrm{MAX}}$, that is

$$
E_{\hat{\mathbf{q}}} \leq E_{\mathrm{MAX}}
$$

8 In this work, the limit to the input energy $E_{\mathrm{MAX}}$ is expressed in terms of the input energy $E_{\mathrm{REF}}$ 9 required by $L$ monopoles in free-field, co-located in the geometrical center of the array, and

10 operating in phase to produce unitary sound pressure at a point located in front of the array at a 11 distance $R$, that is

$$
E_{\mathrm{REF}}=\sum_{\ell=1}^{L}\left(\frac{4 \pi R}{L}\right)^{2}=\frac{(4 \pi R)^{2}}{L} .
$$

12 Assuming that $r_{\ell} \approx R$, which may be the case when the size of the array is compact with respect 13 to the distance between the array and the bright point, then $E_{\mathrm{REF}} \approx E_{\mathrm{DAS}}$, where $E_{\mathrm{DAS}}$ is the 14 input energy of the DAS. However, for a given beamforming method, the above value $E_{\mathrm{REF}}$ may 15 be a restrictive limit for $E_{\mathrm{MAX}}$, especially at low frequencies. Therefore, $E_{\mathrm{MAX}}$ is defined as

$$
E_{\mathrm{MAX}}=\sigma E_{\mathrm{REF}},
$$

16 where $\sigma$ is a positive real-valued factor that is set by the user depending on the configuration 
1 of the array and the control zone. The physical interpretation of the parameter $\sigma$ is that we are

2 allowing the input energy of the compensated filters generated with a beamforming method to be $\sigma$ times higher than the input energy of the DAS.

\subsection{Theoretical considerations on the compensated filters}

The question arises as to the relationship between the compensated filters $\hat{\mathbf{q}}$ and the original filters of the beamforming methods considered in this work. For the (near-field) DAS, the compensated filters $\hat{\mathbf{q}}_{\text {DAS }}$ are identical to the filters qDAS designed with Eq. (4). For what concerns the PM method, in Appendix A the relation is shown between filters of the form $\hat{\mathbf{q}}_{\mathrm{PM}}$ and qPM. For the energy-based methods (e.g., ACM and EDM), if $\mathbf{q}$ is an eigenvector solution to the problems in Eq. (13) or Eq. (16), then $\hat{\mathbf{q}}=W \mathbf{q}$ is also a solution. In general, filters calculated with the ACM and EDM methods have unitary norm (thus constant input energy) regardless of the value of the tunable parameter $\left(\beta_{\mathrm{ACM}}\right.$ or $\alpha$ ) we use for that specific method, that is $\left\|\mathbf{q}_{\mathrm{ACM}}\right\|^{2}=1 \forall \beta_{\mathrm{ACM}}$, where the notation $\forall$ reads "for all values of", and $\left\|\mathbf{q}_{\mathrm{EDM}}\right\|^{2}=1 \forall \alpha$, respectively. However, if we introduce the compensated filters $\hat{\mathbf{q}}$, the tunable parameters can be used to control the input energy to the array. ${ }^{1}$

\subsection{Implementation of the performance constraints}

A number of methods are available in the literature for the calculation of the regularization parameter [27], such as the L-curve or the Generalized Cross Validation, to mention a few. In this work, the values of the tunable parameters are chosen so that the filters designed with a given optimal beamforming method meet the performance constraints set in Section 4. More specifically, for a given beamforming method and the corresponding tunable parameter, the approach is to iteratively increase (or decrease), independently for each frequency, the tunable parameter and to calculate the compensated filters $\hat{\mathbf{q}}$ using Eq. (18) until they meet the constraint on the maximum input energy in Eq. (21). As a result of this procedure, the compensated filters

\footnotetext{
${ }^{1}$ By taking the $\mathrm{ACM}$ as a reference, the input energy of the compensated filters $\hat{\mathbf{q}}_{\mathrm{ACM}}$ for the ACM method can be written as $\left\|\hat{\mathbf{q}}_{\mathrm{ACM}}\right\|^{2}=\left\|W_{\mathrm{ACM}} \mathbf{q}_{\mathrm{ACM}}\right\|^{2}$, where $W_{\mathrm{ACM}}$ is the compensation filter. Using the homogeneity property of the norm, we can write $\left\|\hat{\mathbf{q}}_{\mathrm{ACM}}\right\|^{2}=\left|W_{\mathrm{ACM}}\right|^{2}\left\|\mathbf{q}_{\mathrm{ACM}}\right\|^{2}=\left|W_{\mathrm{ACM}}\right|^{2}$. Since $W_{\mathrm{ACM}}$ is a function of $\beta_{\mathrm{ACM}}$, then $\left\|\hat{\mathbf{q}}_{\mathrm{ACM}}\right\|^{2}$ also depends on $\beta_{\mathrm{ACM}}$. Same considerations apply to the EDM method and the tunable parameter $\alpha$.
} 
Inputs: $\varepsilon_{\beta}, \beta_{\mathrm{MIN}}, E_{\mathrm{MAX}}$

Outputs: $\hat{\mathbf{q}}, \beta$, and $W$.

Initialize $\beta=\beta_{\text {MIN }}$

Calculate $\mathbf{q}(\beta)$ using PM or ACM.

Calculate $\hat{\mathbf{q}}=W \mathbf{q}$

while $E_{\hat{\mathbf{q}}}>E_{\mathrm{MAX}}$ do

$\beta=\beta+\varepsilon_{\beta}$

Calculate $\mathbf{q}(\beta)$ using PM or ACM.

Calculate $\hat{\mathbf{q}}=W \mathbf{q}$

end while

return $\hat{\mathbf{q}}, \beta$, and $W$.

(a)
Inputs: $\varepsilon_{\alpha}, \alpha_{\mathrm{MAX}}, E_{\mathrm{MAX}}$

Outputs: $\hat{\mathbf{q}}, \alpha$, and $W$.

Initialize $\alpha=\alpha_{\mathrm{MAX}}$

Calculate $\mathbf{q}(\alpha)$ using EDM.

Calculate $\hat{\mathbf{q}}=W \mathbf{q}$

while $E_{\hat{\mathbf{q}}}>E_{\mathrm{MAX}} \& \alpha \geq 0$ do

$\alpha=\alpha-\varepsilon_{\alpha}$

Calculate $\mathbf{q}(\alpha)$ using EDM.

Calculate $\hat{\mathbf{q}}=W \mathbf{q}$

end while

return $\hat{\mathbf{q}}, \alpha$, and $W$.

Figure 2: Pseudo-code of the algorithms used for the calculation of the compensated filters for the PM and ACM methods (a) and the EDM method (b).

that meet both performance constraints are obtained. The algorithms, given for a single frequency, are summarized as follows.

- PM and ACM. Starting from a minimum value $\beta_{\mathrm{MIN}}$, the regularization parameter is updated, at each iteration $i$, using the rule $\beta_{i}=\beta_{i-1}+\varepsilon_{\beta}$, where $\varepsilon_{\beta}$ is the step size set by the user. The compensated input signals are then calculated with $\beta_{i}$ and Eq. (8) (PM) or Eq. (15) (ACM). In order to ensure a faster convergence of the algorithm, the domain is chosen as $\left[\beta_{\mathrm{MIN}}, \infty\right)$, where $\beta_{\mathrm{MIN}}$ is set by the user. The iterations stop when the constraint in Eq. (21) is satisfied. The pseudo-code of the algorithm is shown in Fig. 2(a).

- EDM. The EDM tuning parameter $\alpha$ is updated using the rule $\alpha_{i}=\alpha_{i-1}-\varepsilon_{\alpha}$ from $\alpha=\alpha_{\text {MAX }}$ to $\alpha=0$, where $\varepsilon_{\alpha}$ is the step size of the EDM parameter set by the user, and $\alpha_{\text {MAX }}$ is the upper limit to $\alpha$. The pseudo-code of the algorithm is shown in Fig. 2(b). The value $\alpha_{\mathrm{MAX}}$ is calculated with the procedure described in Appendix B using the condition

$$
\alpha_{\mathrm{MAX}}=\alpha:-\delta_{\mathrm{EDM}}<\frac{\partial^{2} \mathrm{AC}(\alpha)}{\partial \alpha^{2}}<\delta_{\mathrm{EDM}}
$$

where $\delta_{\mathrm{EDM}}$ is a small threshold set by the user and $\partial^{2} \mathrm{AC}(\alpha) / \partial \alpha^{2}$ is the second partial derivative of the acoustic contrast with respect to $\alpha$.

- DAS. Filters with the DAS method have been designed by using Eq. (4). 


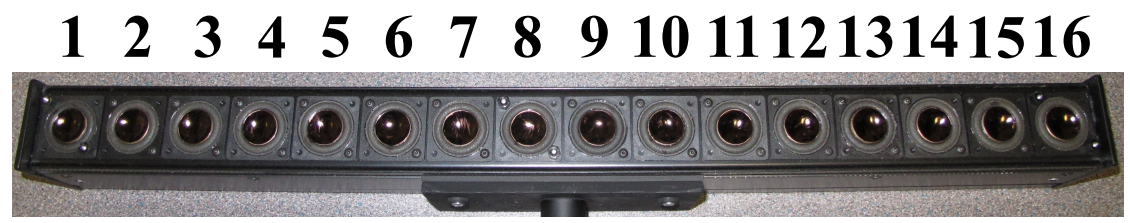

Figure 3: (Color online) The 16-channel linear loudspeaker array prototype and the loudspeaker numbering.

\section{Validation of the proposed strategy for comparison of beamforming methods}

\subsection{Linear array prototype and definition of the beamforming problem}

The loudspeaker array used for the simulations and the experiments in this work is shown in

4 Fig. 3. It consists of $L=16$ electrodynamic drivers arranged on a line and spaced at a distance $\Delta=$ $53.86 \mathrm{~cm}$. The size of the array is approximately $A=0.62 \mathrm{~m}$. The coordinate of the $\ell$-th loudspeaker 6 of the array is $\mathbf{y}_{\ell}^{T}=[(\ell-8.5) \Delta, 0,0], \ell \in[1, \ldots L]$. The beamforming problem for the linear array prototype consists of $M=37$ control points uniformly arranged on a semi-circle of radius $R=3 \mathrm{~m}$. The coordinate of the $m$-th control point is $\mathbf{x}_{m}^{T}=[R \cos (\Delta \theta(m-1)), R \sin (\Delta \theta(m-1))]$, $m=1, \cdots, M$, where $\Delta \theta=5^{\circ}$. With reference to Fig. 4(a) and Fig. 4(b), two sound beams are considered, one steered to the front and one to the side of the array, respectively. The angular coordinates of the bright points given in polar coordinates for the front and the side beams are $\theta_{B 1}=90^{\circ}$ (control point \#19) and $\theta_{B 2}=50^{\circ}$ (control point \#11), respectively. For both beams, 3 the dark area consists of $M_{D}=36$ control points. For a beam steered to a control point of angular 14 coordinate $\theta_{0}$, the upper frequency limit $f_{\mathrm{a}}$ due to the spatial aliasing is [21]

$$
f_{\mathrm{a}}\left(\theta_{0}\right)=\frac{c}{\Delta\left(1+\left|\cos \left(\theta_{0}\right)\right|\right)},
$$

which gives, for each beam, $f_{\mathrm{a} 1}=f_{\mathrm{a}}\left(\theta_{B 1}\right) \approx 8.89 \mathrm{kHz}$ and $f_{\mathrm{a} 2}=f_{\mathrm{a}}\left(\theta_{B 2}\right) \approx 5.4 \mathrm{kHz}$, respectively. Results for each beam are likely to be affected by spatial aliasing at frequencies above those two limits.

\subsection{Design of the compensated filters}

The compensated filters for the two beamforming problems in Fig. 4(a) and Fig. 4(b) were designed using the considered beamforming methods and the analytical transfer function $\mathbf{Z}$. The 


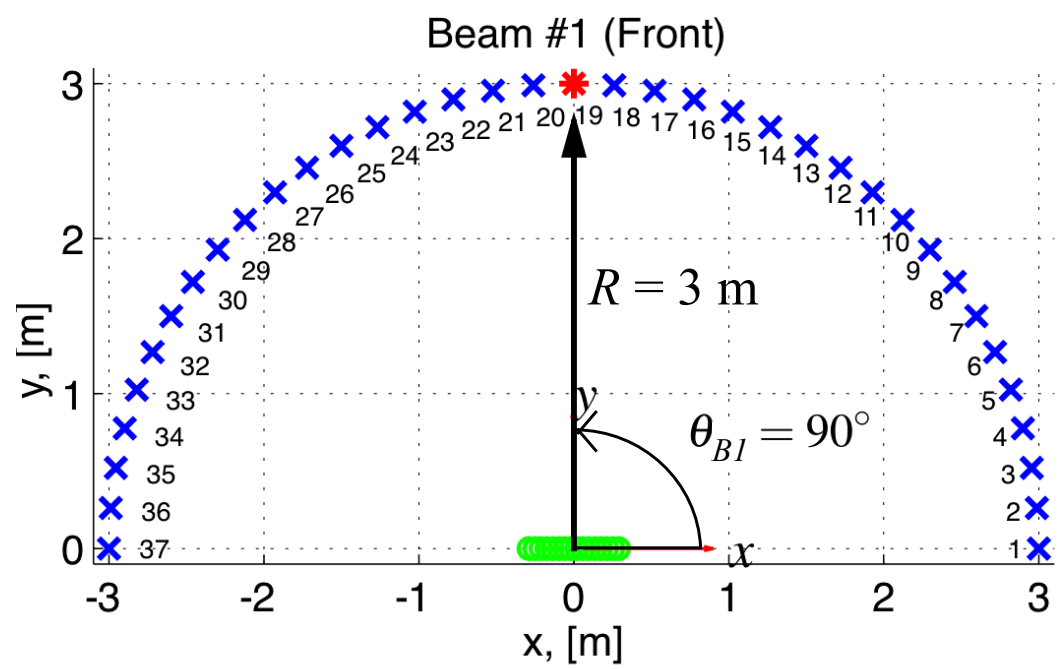

O Source of the array $*$ Bright point $\times$ Dark point

(a)

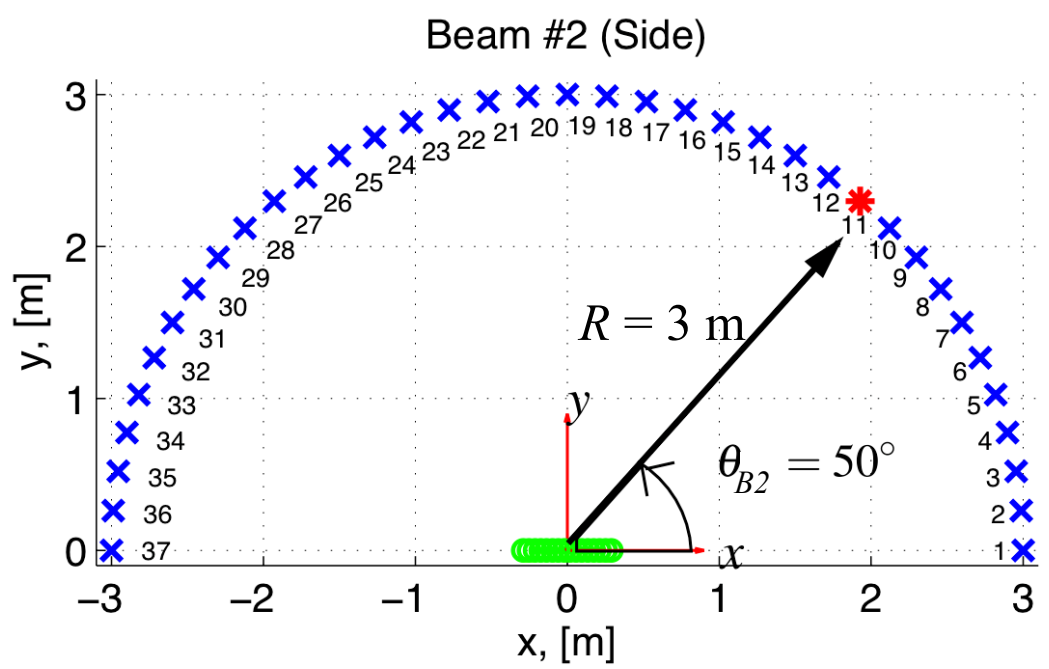

O Source of the array $*$ Bright point $\times$ Dark point

(b)

Figure 4: Beamforming problems for the linear array. Configurations of the front (a) and side (b) beams.

$\sigma$ factor for the limit on the input energy to the array was arbitrarily set to $\sigma=25$, which leads to

2 a value of $E_{\mathrm{MAX}}$ of 


$$
E_{\mathrm{MAX}}(\mathrm{dB})=14 \mathrm{~dB} \text { re } E_{\mathrm{DAS}}
$$

1 Furthermore, $E_{\mathrm{MAX}}$ is chosen to be constant over frequency. Smaller values of $\sigma$ yield lower values of $E_{\mathrm{MAX}}$ which result into higher values of the regularization parameters which, in turn, may results in reduced directivity performance. We have empirically chosen $\sigma=25$, which is 2 dB higher than $10 \log _{10}(L)=12(\mathrm{~dB})$, and we have assumed that the array can be driven with input signals whose energy does not exceed $E_{\mathrm{MAX}}$. The implications of such a choice of $\sigma$ are discussed in the next sections. The values of the parameters for the algorithms for the calculation of the filters for the PM method are $\varepsilon_{\beta}^{\mathrm{PM}}=10^{-6}$ and $\beta_{\mathrm{MIN}}^{\mathrm{PM}}=10^{-20}$. Those used for the ACM method are $\varepsilon_{\beta}^{\mathrm{ACM}}=10^{-9}$ and $\beta_{\mathrm{MIN}}^{\mathrm{ACM}}=10^{-20}$. The step size for the calculation of the tunable parameter for the EDM method is $\varepsilon_{\alpha}=10^{-3}$. The values of $\delta_{\mathrm{EDM}}=0.01$ and the upper limit to $\alpha_{\mathrm{MAX}}$ is 200 . The value of $\alpha_{\mathrm{MAX}}$ calculated as described in Appendix B have been used for the design of the compensated input signals with the EDM method for both the front and the side beams. The filter design was performed using a sampling frequency of $f_{s}=48 \mathrm{kHz}$ and the number of filter coefficients is $N_{\mathrm{FFT}}=8192$. A modeling delay of $\Delta t=N_{\mathrm{FFT}} /\left(2 f_{s}\right)$ was applied to all the designed filters.

\subsection{Simulations and Experiments}

5.3.1. Experimental setup

The responses of the array driven with the designed filters were measured in the ISVR Anechoic Chamber, that has dimensions $[9.15 \times 9.15 \times 7.32] \mathrm{m}^{3}$ and provides for an excellent approximation of free-field condition above $80 \mathrm{~Hz}$ [22]. Furthermore, the TF matrix $\mathbf{H}$, whose $(m, \ell)$-th element, indicated by $H_{m, \ell}$, is the response between the $\ell$-th transducer of the array and the $m$-th control point measured under free-field conditions. Matrix $\mathbf{H}$ was measured to evaluate the matching of the TFs of the various transducers of the array as discussed in the next subsection. 23 The temperature measured in the anechoic chamber was $20^{\circ} \mathrm{C}$, that leads to an estimated value of the speed of sound $c=343 \mathrm{~ms}^{-1}$. The details of the measurements are described next. The linear loudspeaker array and an omni-directional microphone (B\&K 4189) were installed in the anechoic chamber as shown in Fig. 5(a) and placed at $2 \mathrm{~m}$ above the ground. The distance between 
1 block diagram shown in Fig. 5(b), the hardware chain used for both experiments in the ISVR 2 anechoic chamber is as follows. The loudspeaker array was placed on top of a turntable (Outline ET250-3D) which was connected to the computer via an Ethernet cable. The microphone was connected to the NEXUS signal conditioner and the transducers of the loudspeaker array were connected to a set of custom-built amplifiers. The output of the microphone signal conditioner and the inputs of the loudspeaker amplifier were connected to a digital sound-card interface (Ferrofish A16 MKII). The sound-card was connected through a MADI cable to a MADI to USB converter (RME MADIFace) and the latter was connected to a laptop computer through a USB cable. The laptop computer was used to reproduce the signals with the loudspeaker array and to record the signal captured by the microphone. The elements of $\mathbf{H}$ were estimated using the so-called H1 estimator [23] from the recordings of the response between each loudspeaker of the array and the microphone to a broadband signal (white noise of unitary standard deviation) of duration $6 \mathrm{~s}$. This procedure was repeated 37 times by rotating the turntable every $5^{\circ}$ in counter-clockwise direction from $0^{\circ}$ to $180^{\circ}$. The same setup was used for the estimation of the responses of the array driven by the designed filters.

\subsubsection{Electro-acoustical analysis of the array}

As previously stated, the filter design process is performed using an analytical model (i.e., ideal monopoles) for the radiation of the transducers of the array (i.e., matrix $\mathbf{Z}$ ). However, the responses of the real speakers differ from the responses of the monopole sources (that have flat magnitude FRF as a function of frequency) as shown in Fig. 6(a), were the measured magnitude FRFs of loudspeaker $\# 8$ at $50^{\circ}$ and $90^{\circ}$ (i.e., the angular coordinates of the control points \#11 and $\# 19$, respectively) are plotted. Most noticeably, between $2 \mathrm{kHz}$ and $3 \mathrm{kHz}$ there is a "jump" in the responses that is a characteristic of the transducers installed in the array. The implicit assumption made in the filter design process is that the monopoles are identical (matched) between each other. The same cannot be said for the actual speakers of the array, whose responses are likely to differ between each other due to a number of factors, such as the diffraction of the array enclosure. In this section, we evaluate at which frequencies the actual speakers are mismatched. For a given angle (control point), we evaluate the standard deviation of the magnitude squared of the measured and analytical transfer functions both normalized by a given reference response, that is $\mathbf{H} / \tilde{H}$ 

$\zeta_{\mathrm{msrd}}$, respectively, are calculated as

$$
\begin{aligned}
& \zeta_{\text {anlt }}\left(\mathbf{x}_{m}\right)=\sqrt{\frac{1}{L} \sum_{\ell=1}^{L}\left(\left|\frac{Z_{m, \ell}}{\tilde{Z}}\right|^{2}-\frac{1}{L} \sum_{\ell=1}^{L}\left|\frac{Z_{m, \ell}}{\tilde{Z}}\right|^{2}\right)^{2}}, \\
& \zeta_{\mathrm{msrd}}\left(\mathbf{x}_{m}\right)=\sqrt{\frac{1}{L} \sum_{\ell=1}^{L}\left(\left|\frac{H_{m, \ell}}{\tilde{H}}\right|^{2}-\frac{1}{L} \sum_{\ell=1}^{L}\left|\frac{H_{m, \ell}}{\tilde{H}}\right|^{2}\right)^{2}} .
\end{aligned}
$$

4 The standard deviation in the case of monopole sources, $\zeta_{\text {anlt }}$, can be calculated analytically by 5 means of the following formula

$$
\zeta_{\text {anlt }}\left(\mathbf{x}_{m}\right)=\sqrt{\frac{\tilde{r}^{4}}{L} \sum_{\ell=1}^{L}\left(\frac{1}{r_{m, \ell}^{2}}-\frac{1}{L} \sum_{\ell=1}^{L} \frac{1}{r_{m, \ell}^{2}}\right)^{2}},
$$

where $\tilde{r}$ is the distance between monopole $\# 8$ and the control point at $90^{\circ}$, and $r_{m, \ell}=\left\|\mathbf{x}_{m}-\mathbf{y}_{\ell}\right\|$. If the FRFs of the sources are matched, the standard deviation is constant over frequency. Fig. 6(b) shows the standard deviations of the transfer functions at microphone positions $50^{\circ}$ and $90^{\circ}$, respectively. As expected, the standard deviations in the case of monopole sources are constant over frequency and their values, calculated with Eq. (29), are equal to $\zeta_{\text {anlt }}\left(\mathbf{x}_{11}\right)=0.0761$ and $\zeta_{\text {anlt }}\left(\mathbf{x}_{19}\right)=0.0031$, respectively. Conversely, the peaks in the standard deviation of the measured responses at $955 \mathrm{~Hz}$ and $1676 \mathrm{~Hz}$ indicate that, around those frequencies, the measured responses differ from the mean value of $\left|H_{m, \ell} / \tilde{H}\right|^{2}$. In other words, the FRFs of the loudspeakers are likely to be mismatched between each other. These two frequencies (and the frequencies nearby) are critical for the robustness of the system and this is further discussed in the next sections.

\subsubsection{Performance parameters}

System performance of the linear array driven with the compensated filters $\hat{\mathbf{q}}$ designed in the previous section are evaluated using simulated filter responses $\tilde{\mathbf{p}}=\mathbf{Z} \hat{\mathbf{q}}$ and with the responses of the filters measured in the anechoic chamber in terms of FRF of the pressure field at the bright point, Acoustic Contrast AC (Eq. (12)), and Array Efficiency $(A E)$.

Let $p_{B}$ be the sound pressure measured at the bright point due to the array driven by the 




(a)

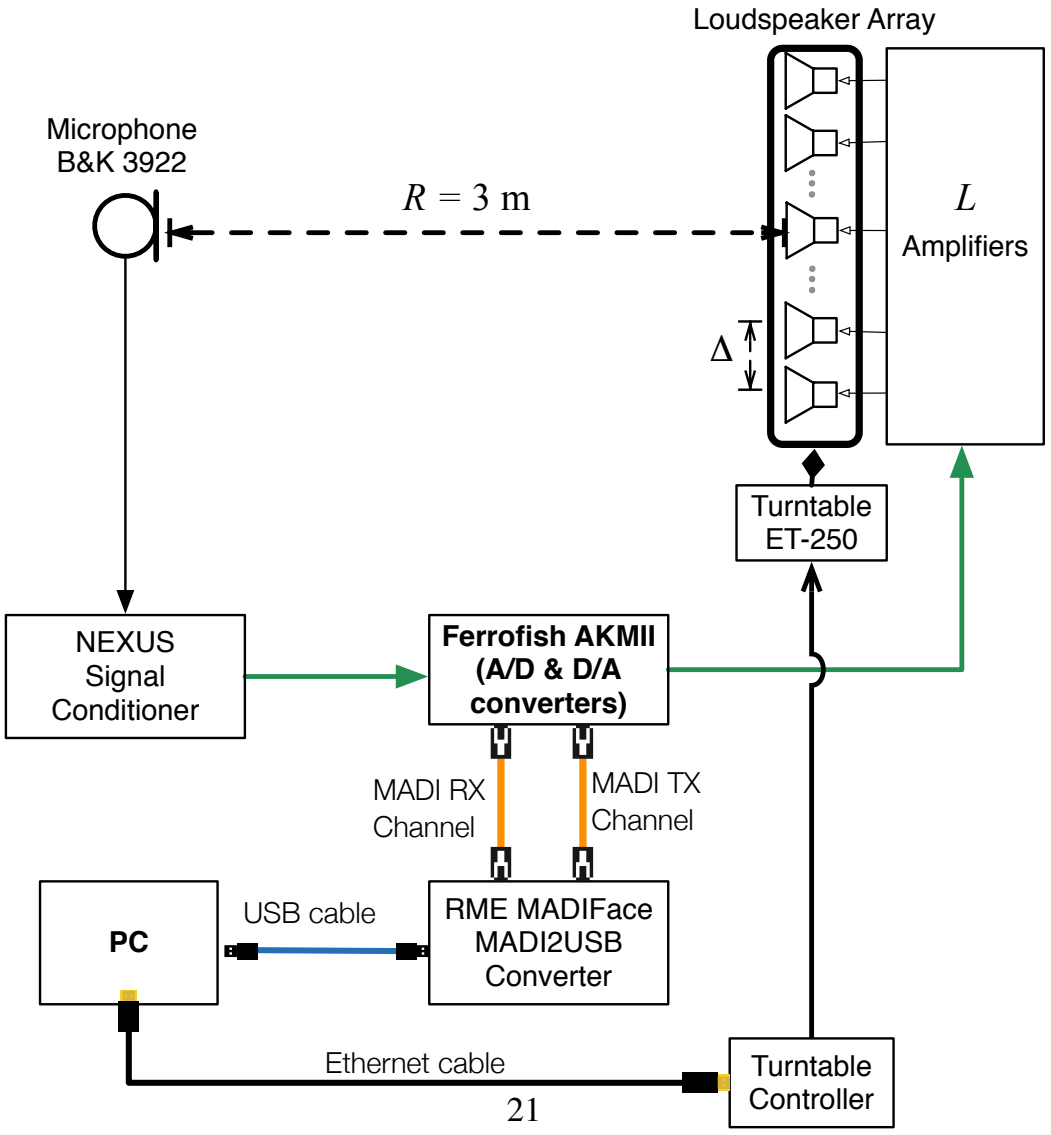

(b)

Figure 5: (Color online) The linear loudspeaker array prototype and microphone installed in the ISVR anechoic chamber (a) and block diagram of the experimental set-up (b). 


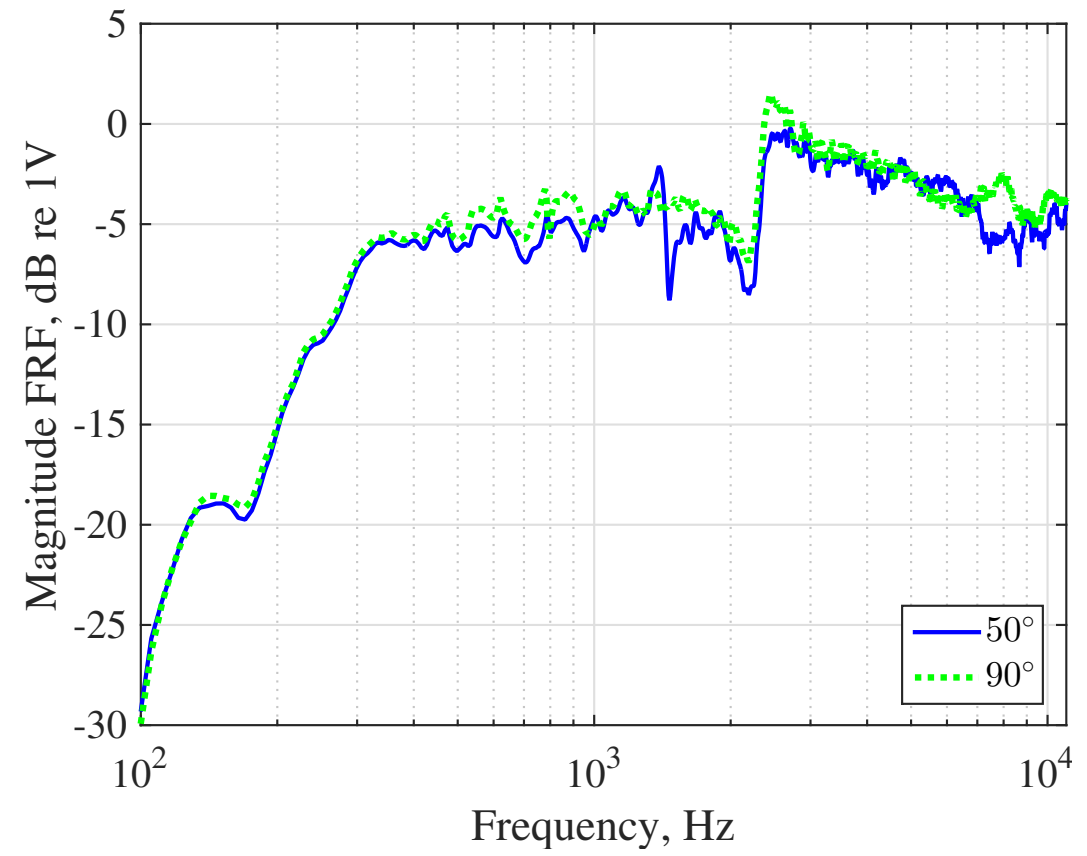

(a)

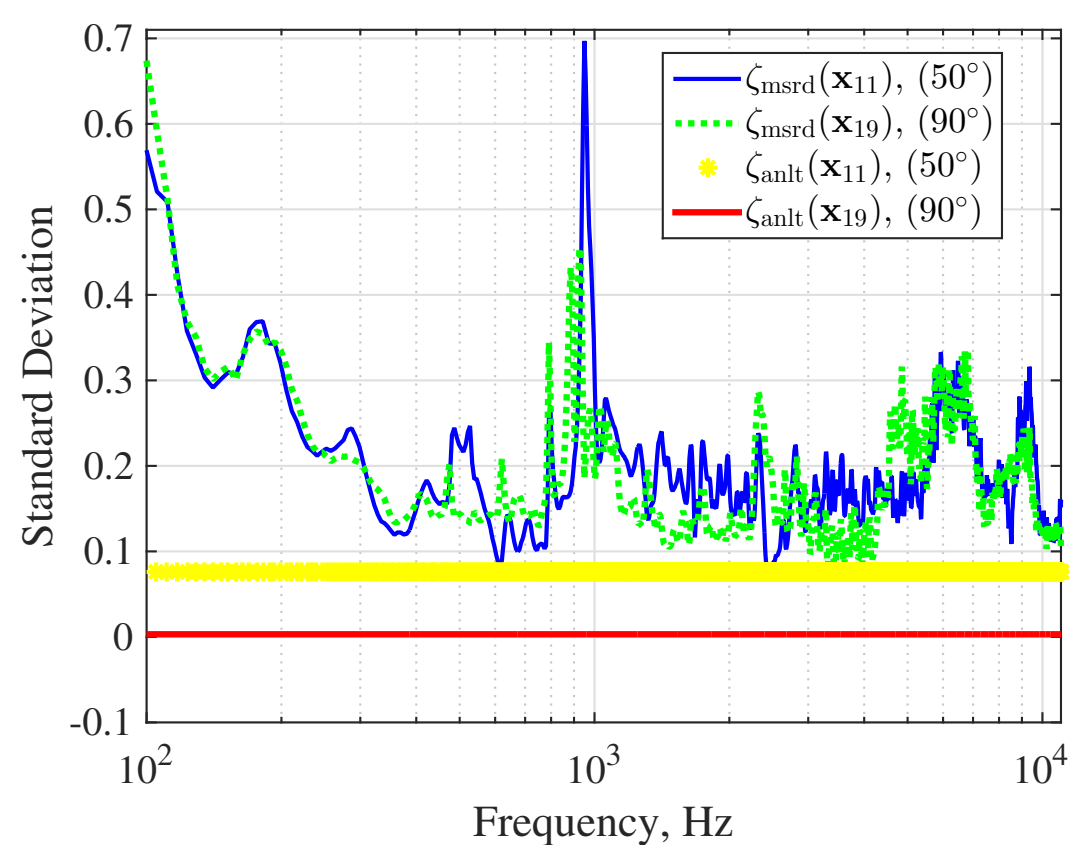

(b)

Figure 6: Electro-acoustical analysis of the linear loudspeaker array. Magnitude FRF of the measured transfer functions between loudspeaker \#8 and two microphone positions (a). Standard deviation of measured and analytical responses at two microphone positions (b). 
1 input signals $\hat{\mathbf{q}}$ whose input energy is $E_{\hat{\mathbf{q}}}$. In general, the source configuration that requires the 2 lowest input energy $E_{\mathrm{LSRC}}$ to reproduce $p_{B}$ at the bright point consists of $L$ sources (either ideal monopoles of real loudspeakers), co-located at the acoustic center of the array and operating in phase. The Array Efficiency $(A E)$ is defined as the ratio between $E_{\mathrm{LSRC}}$ and the input energy $E_{\hat{\mathbf{q}}}$, that is

$$
A E=10 \log _{10}\left(\frac{E_{\mathrm{LSRC}}}{E_{\hat{\mathbf{q}}}}\right)=10 \log _{10}\left(\frac{\left|p_{B}\right|^{2}}{L\left|Z_{\mathrm{REF}}\right|^{2} E_{\hat{\mathbf{q}}}}\right),
$$

where $Z_{\mathrm{REF}}$ is the reference transfer function between a single source and the bright point. For the simulations and experiments, the reference transfer functions have been chosen as $Z_{\mathrm{REF}}=\tilde{Z}$ and

8 $Z_{\mathrm{REF}}=\tilde{H}$, respectively, where $\tilde{Z} \approx e^{-j(\omega / c) R} / 4 \pi R$ is the reference transfer function calculated using the monopole model for loudspeaker \# 8 and the control point \# 19. The AE, whose range 10 is $[-\infty, 0] \mathrm{dB}$, indicates the similarity (in terms of input energy) of $\hat{\mathbf{q}}$ with respect to the most efficient source configuration. For a given $\hat{\mathbf{q}}$, higher values of $A E$ are desirable.

\subsection{Results for the front beam}

The filters designed with optimal beamforming methods for the front beam meet the constraint in Eq. (21) as shown in Fig. 7(a), that shows the input energy $E_{\hat{\mathbf{q}}}$ required by the designed filters. The input energy is identical for all the optimal methods across the whole frequency range up to the aliasing frequency. Furthermore, the input energy is equal to $E_{\mathrm{MAX}}$ (i.e., $10 \log _{10} \sigma=14$ $\mathrm{dB}$ ) at low frequencies (below $f_{1} \approx 3.2 \mathrm{kHz}$ ), that is the frequency region where the array is small compared to the wavelength and the filters need high input energy to control the sound field. The input energy of the optimal methods drops from $E_{\mathrm{MAX}}$ to that of the DAS in the transition band between $f_{1}$ and $f_{2} \approx 4 \mathrm{kHz}$, and it keeps constant up to $f_{3} \approx 6.5 \mathrm{kHz}$. With reference to Fig. 7(b), the same trend (as a function of frequency) as per the input energy is observed in the magnitude FRF of the compensated filters for the PM, ACM, and EDM methods for loudspeaker \#5, that are denoted with $\hat{q}_{\mathrm{PM}, 5}, \hat{q}_{\mathrm{ACM}, 5}$, and $\hat{q}_{\mathrm{EDM}, 5}$, respectively. The compensated filters designed with the optimal methods are all identical up to the aliasing frequency. Above the aliasing frequency, the PM and ACM are still identical, whilst the EDM differs. Note that both the input energy and the FRFs of the filters of the optimal methods (see subplots of Fig. 7) are identical between each other at frequencies below $1 \mathrm{kHz}$ and results below this frequency have not been included in the 
1 plots in Fig. 7.

With reference to the calculated tunable parameters for the PM, ACM, and EDM methods shown in Fig. 8, the drops in the frequency responses of both the input energy (see Fig. 7(a)) and the filters (see Fig. 7(b)) observed at $f_{1}$ happen if the regularization parameters for the compensated PM and ACM methods (see Fig. 8(a)) and the parameter $\alpha$ for the EDM method (see Fig. 8(b)) become equal to $\beta_{\mathrm{MIN}}$ and $\alpha_{\mathrm{MAX}}$, respectively. The rapid variations of $\alpha$ (Fig. 8(b)) above the aliasing frequency are reflected in the input energy of the EDM method (Fig. 7(a)). Most noticeably, by introducing the constraint in Eq. (21), the calculated $\alpha$ is lower than $\alpha_{\mathrm{MAX}}$ at frequencies below $f_{1}$. Beside the analysis of the tunable parameters and their relation to the above-mentioned frequency regions, the magnitude and phase responses of the compensation filters $W$ for the optimal methods shown in the subplots of Fig. 9 provide some further insight on the characteristics of the optimal methods. For example, the compensation filter $W_{\mathrm{PM}}$ for the PM method is a frequency-dependent gain (equalizer) with phase response equal to zero. This highlights the fact that the PM method, in order to satisfy the constraint in Eq. (20), needs to "boost" low frequencies. Below the aliasing frequency, the compensation filters $W_{\mathrm{ACM}}$ and $W_{\mathrm{EDM}}$ for the ACM and the EDM methods, respectively, can be regarded as shelving filters with steep transition at $f_{1}$ and linear phase response. The latter information is important as it denotes that filters designed with energy-based methods (that are phase ambiguous) need compensation of their phase response in order to meet the constraint in Eq. (20). Due to the fact that $\hat{\mathbf{q}}_{\mathrm{DAS}}=\mathbf{q}_{\mathrm{DAS}}$ (see Section 4.1), $W_{\text {DAS }}=1$ for all frequencies, where $W_{\text {DAS }}$ is the compensation filter for the DAS.

The sharp "jump" in the magnitude FRF of the filters shown in Fig. 7(b) suggests that the filters may be ringing at $f_{1}$. Let us consider the impulse responses (IR) of the filters for loudspeaker \#5 for the PM and EDM methods that are obtained by inverse Fourier transform of $\hat{q}_{\mathrm{PM}, 5}$ and $\hat{q}_{\mathrm{EDM}, 5}$. Portions of their time histories (from sample \# 2800 to \# 5600) are shown in Fig. 10(a) (PM) and Fig. 10(b) (EDM), respectively. The IR of the ACM method has not been included because it is identical to that of the PM. Most of the energy of the IRs in the subplots of Fig. 10 is concentrated around the largest peaks and the number of filter coefficients $N_{\mathrm{FFT}}$ is enough to represent the IRs. However, the time domain support of the IR of $\hat{q}_{\mathrm{EDM}, 5}$ is wider than that of the IR $\hat{q}_{\mathrm{PM}, 5}$. This may be due to the rapid variations of $\alpha$ at high frequencies (Fig. 8(b)) 


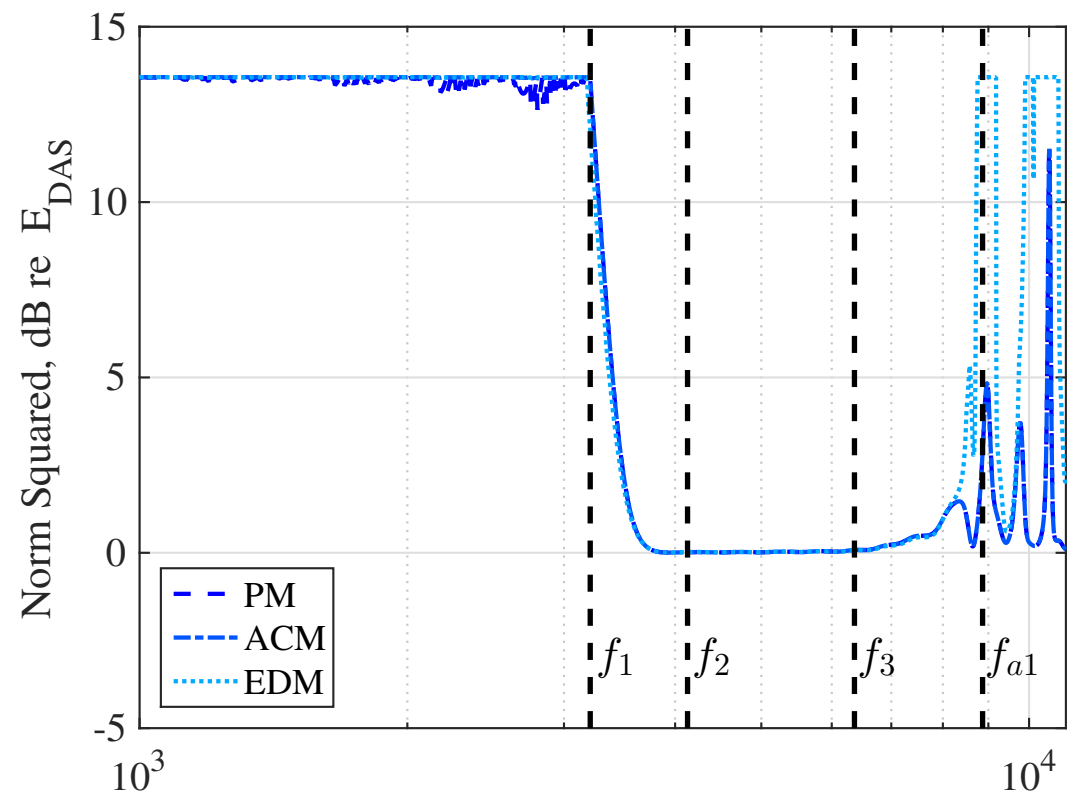

Frequency, $\mathrm{Hz}$

(a)

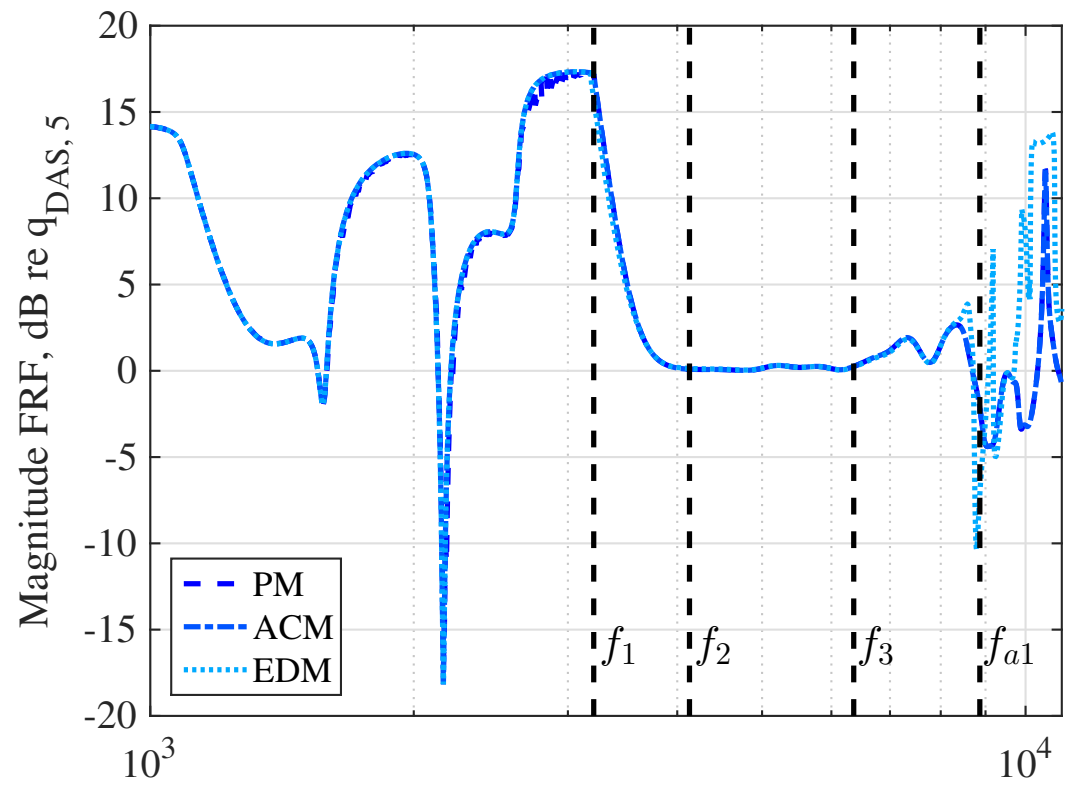

Frequency, $\mathrm{Hz}$

(b)

Figure 7: Front beam. Input energy required by the designed filters (a). Magnitude FRF of the optimal filters (PM, ACM, EDM) for loudspeaker \#5 normalized by the response of the DAS filter for loudspeaker \#5 (b). The vertical dashed lines are the frequencies indicating the three regions for the front beam. 
1 that reflect in the FRF of $\hat{q}_{\text {EDM.5. }}$. Both the IRs show pre- and post-ringing and these time domain artifacts may be introduced by regularization [26].

In the light of the analysis of the IRs of the filters and the measured responses of the loudspeakers (see Fig. 6(a)), the FRF of the designed beamforming filters (for both beams) have been pre-filtered with a bandpass filter. The latter was chosen as a Butterworth band-pass filter of order 3 with cut-on frequency $100 \mathrm{~Hz}$ and cut-off frequency of $9 \mathrm{kHz}$. In order not to modify the phase response of the original beamforming filters, the latter were filtered with the Butterworth filter by means of the so-called zero-phase filtering technique [24]. With reference to Fig. 6(a), the cut-on frequency was chosen in order to avoid driving the loudspeakers in the frequency region where they are less efficient and they are likely to produce distorted output (due to the fact that the input energy of the designed filters is high at low frequencies). The cut-off frequency was chosen to be slightly higher than the highest aliasing frequency (i.e., $f_{\mathrm{a} 1}$ ) because above that frequency the designed filters show rapid and erratic variations as well as spikes in their frequency responses (Fig. 7(b)). These variations may be potentially dangerous for the speakers, and, hence, they were reduced by means of the bandpass filter.

The simulated and measured array efficiency shown in the subplots of Fig. 11 provide information about the reproduced pressure at the bright point and the input energy, that have been constrained to be constant. The $14 \mathrm{~dB}$ range difference between the input energy (see Fig. 7(a)) of optimal methods and DAS is also noticed in the simulated array efficiency in Fig. 11(a) and, on average, in the measured efficiency as well (see Fig. 11(b)). Above $f_{2}$ and below $f_{3}$, the optimal methods are as efficient as the DAS. As previously stated, the range of the array efficiency is $A E \in[-\infty, 0] \mathrm{dB}$. However, at some frequencies, the measured array efficiency (Fig. 11(b)) is slightly higher than zero. This overestimation may be due to inaccuracies in the measurements of the transfer functions and filter responses.

The simulated and measured acoustic contrast of the designed filters for the front beam shown in the subplots of Fig. 12 reveal, as expected, that directivity performance of the optimal methods are identical in the frequency range up to the aliasing frequency. In the simulations (see Fig. 12(a)), where we use the same TFs (i.e., $\mathbf{Z}$ ) for the filter design and evaluation of performance, the AC provided by the optimal methods is higher than that provided by the DAS at frequencies below $f_{2}$. Above $f_{2}$, where the regularization is equal to $\beta=\beta_{\mathrm{MIN}}$ and $\alpha=\alpha_{\mathrm{MAX}}$, all the methods have 


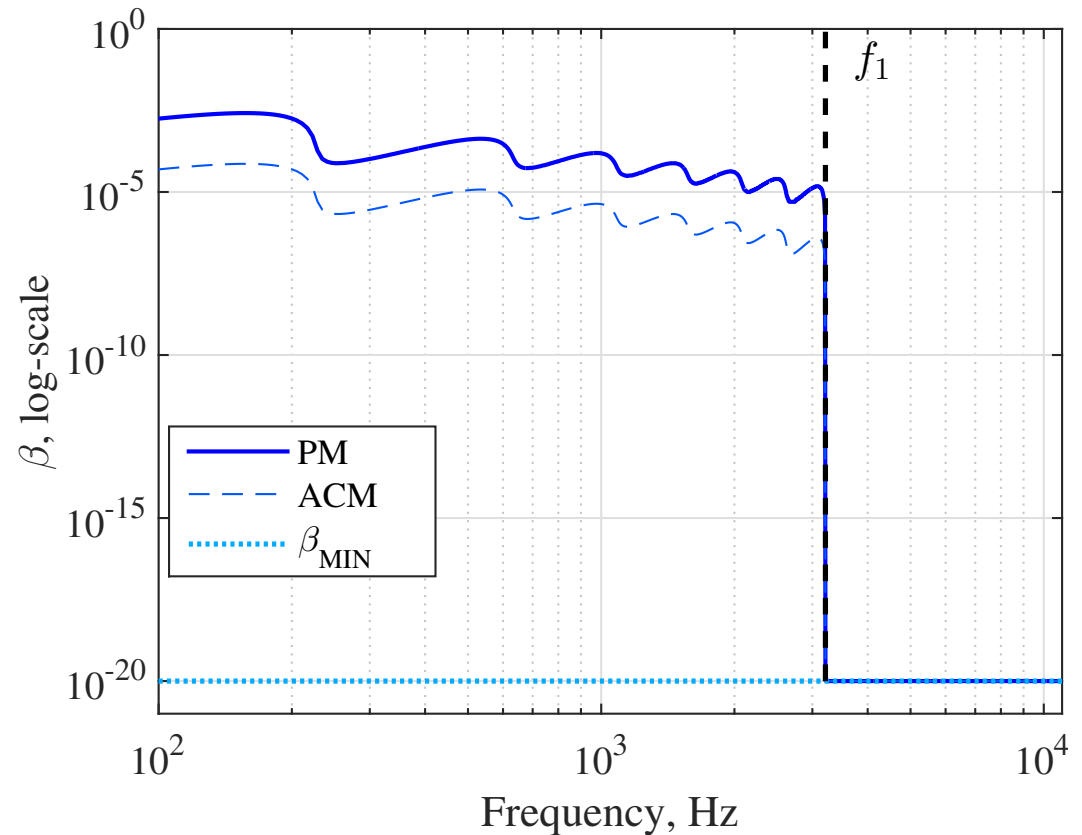

(a)

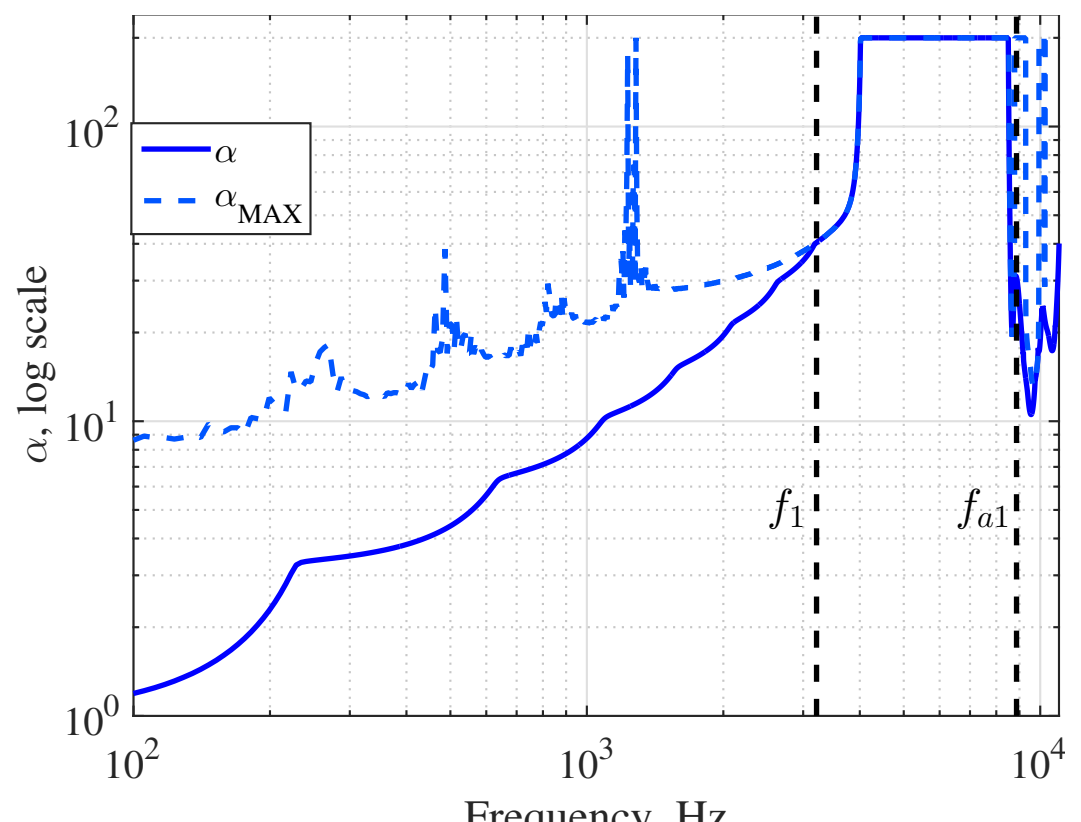

(b)

Figure 8: Front beam. Calculated regularization parameters for PM and ACM method (a) and $\alpha$ for the EDM method (b). 


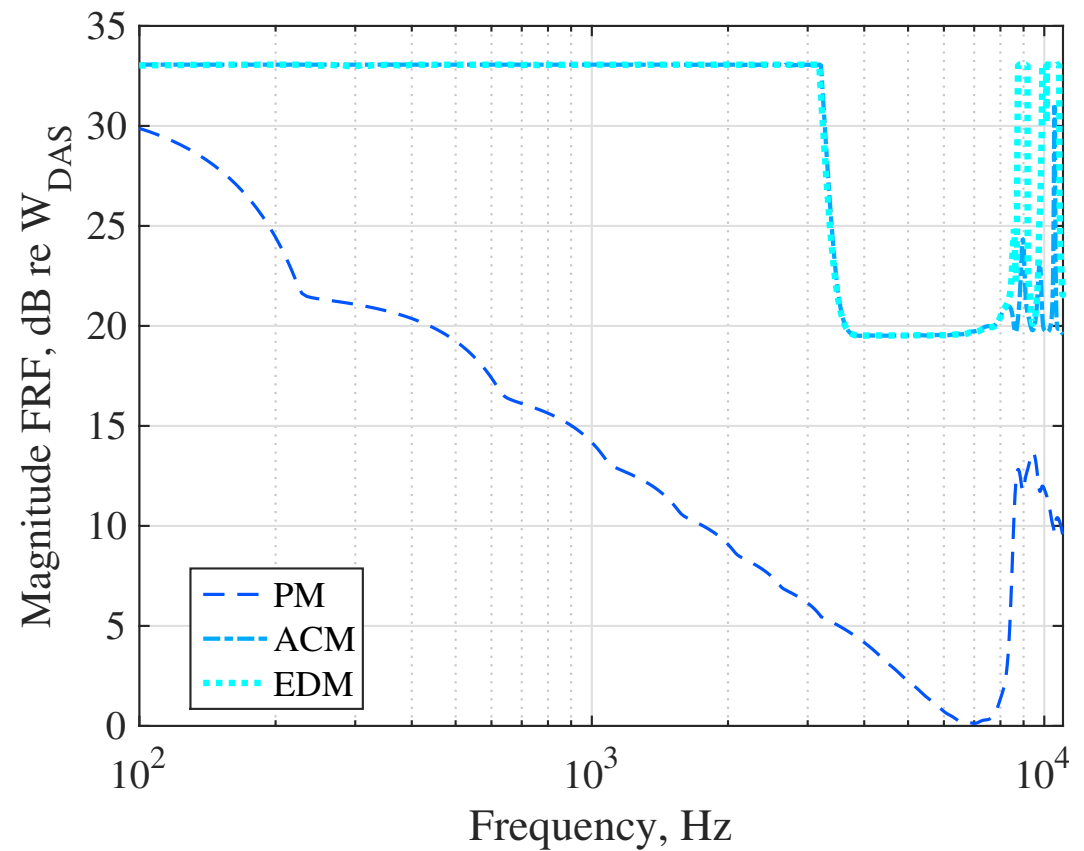

(a)

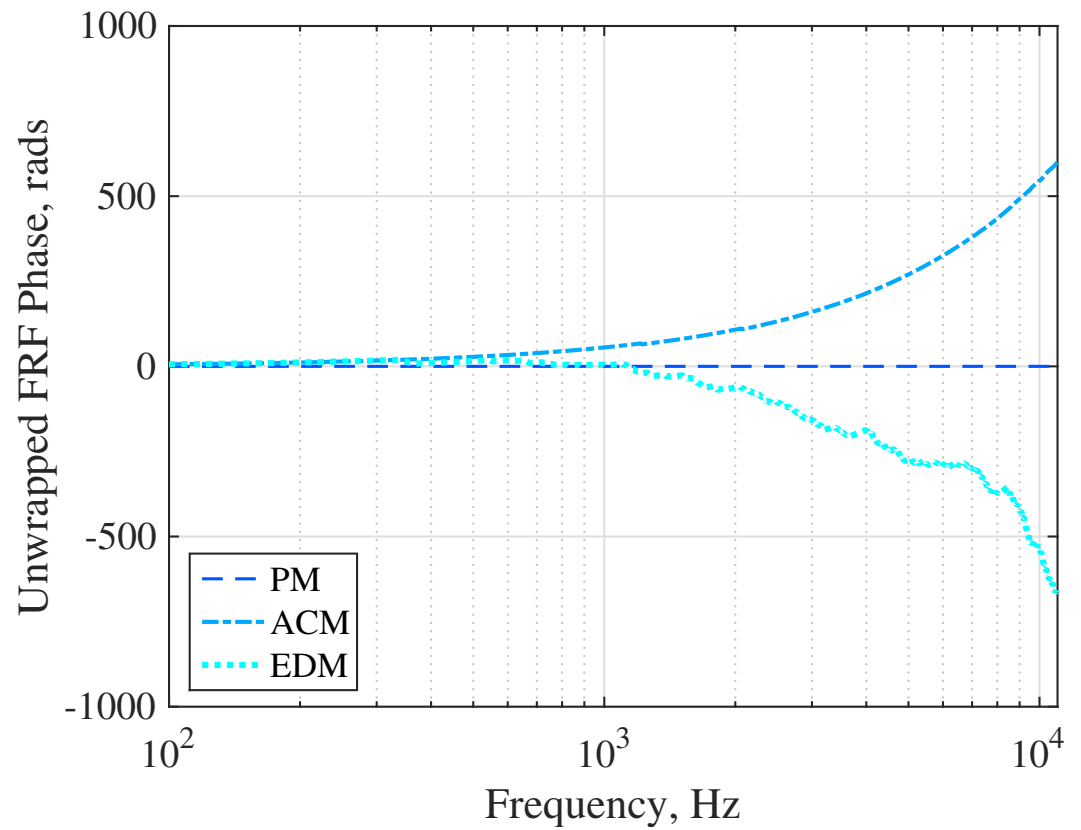

(b)

Figure 9: Front beam. FRFs of the compensation filters $W / W_{\text {DAS }}$ for the optimal methods. Magnitude (a) and phase (b) responses. 


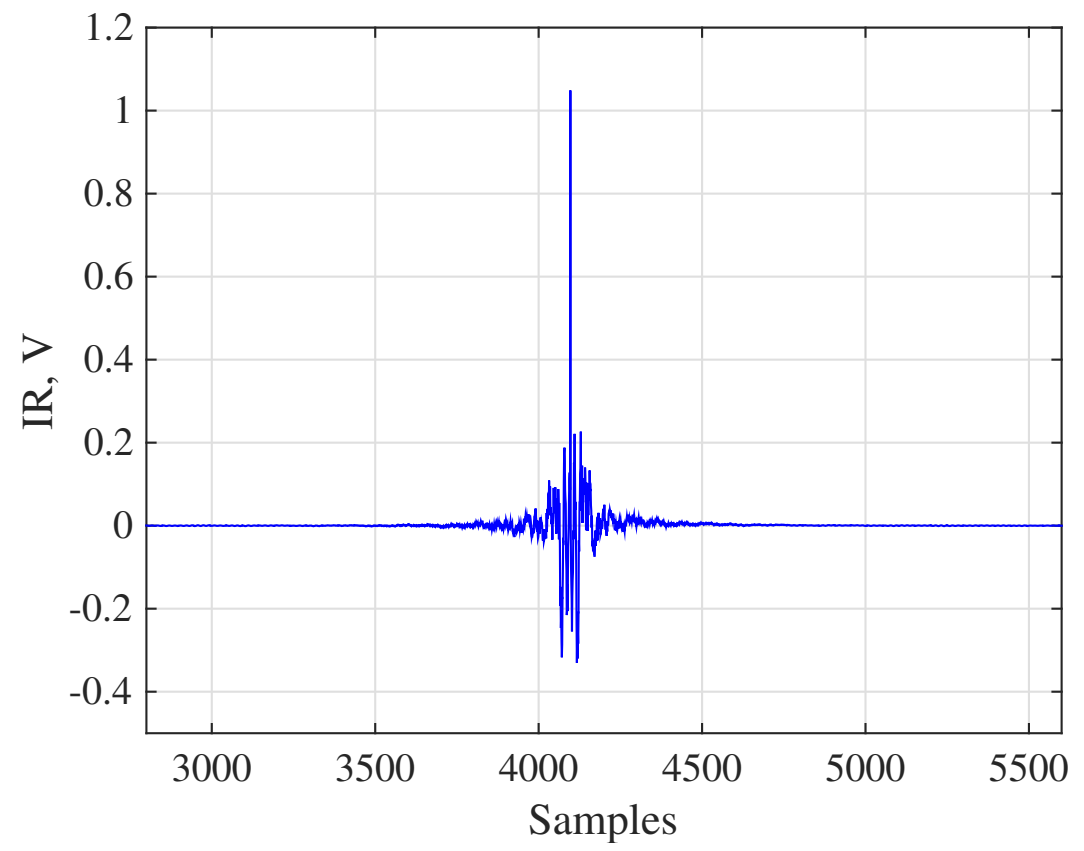

(a)

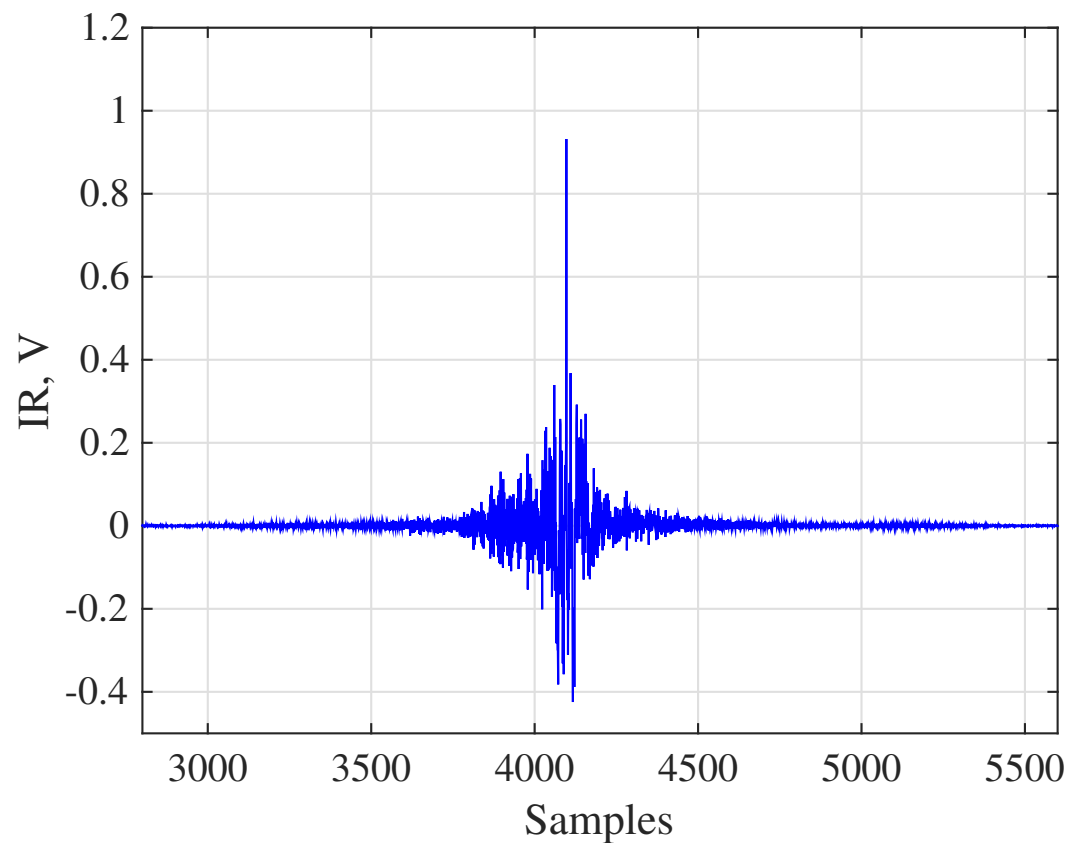

(b)

Figure 10: Front beam. Impulse responses of the filters for loudspeaker \#5 for the PM (a) and EDM (b) methods. The FRFs of both filters is shown in Fig. 7(b). 


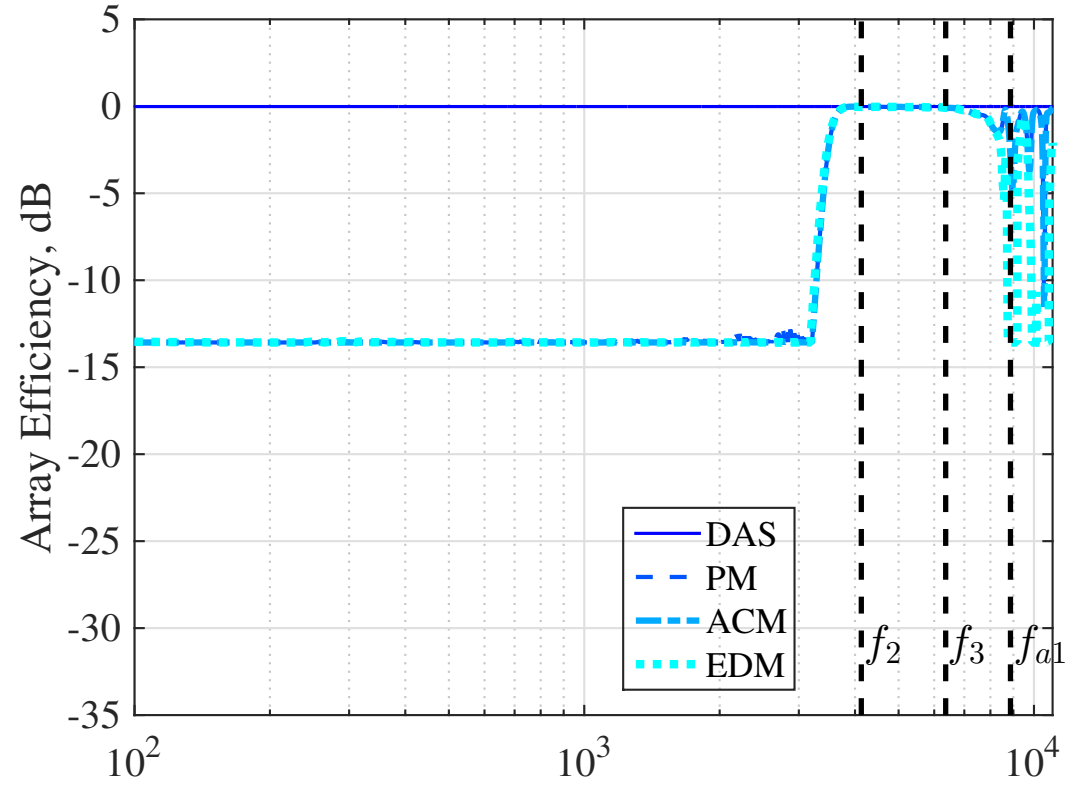

Frequency, $\mathrm{Hz}$

(a)

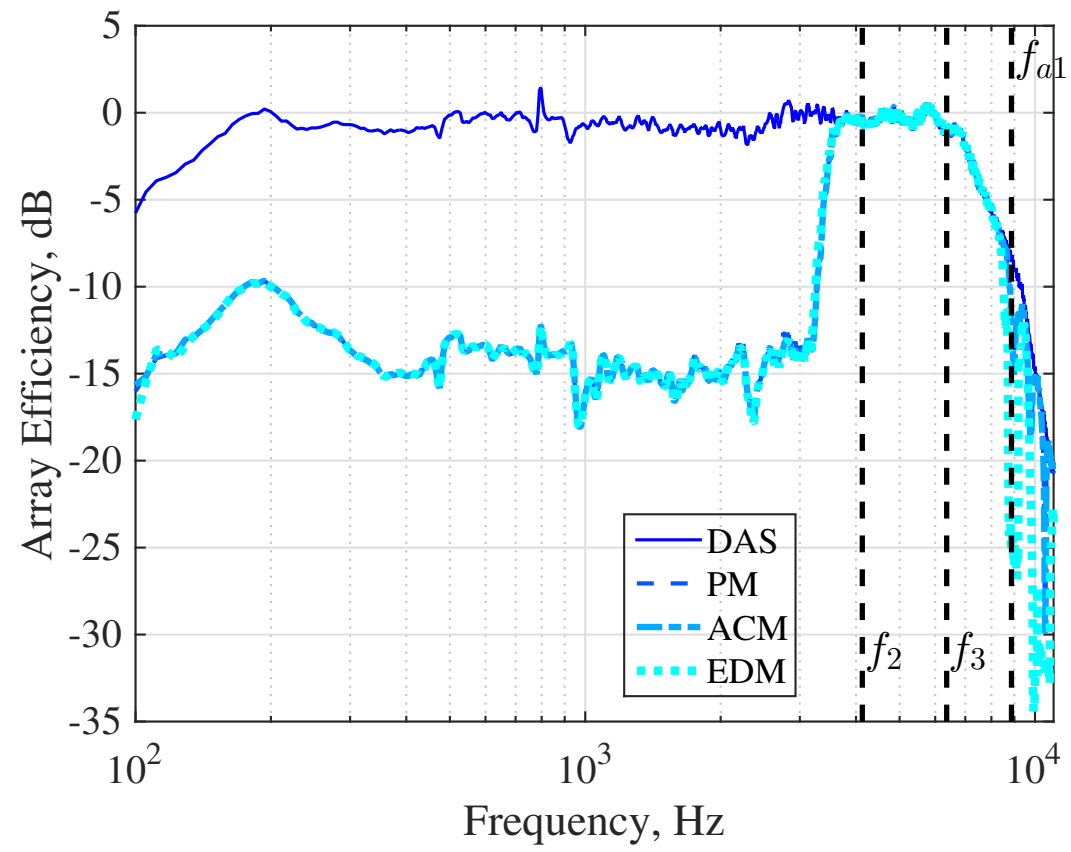

(b)

Figure 11: Simulated (a) and measured (b) array efficiency for the front beam due to filters designed with four beamforming methods. 


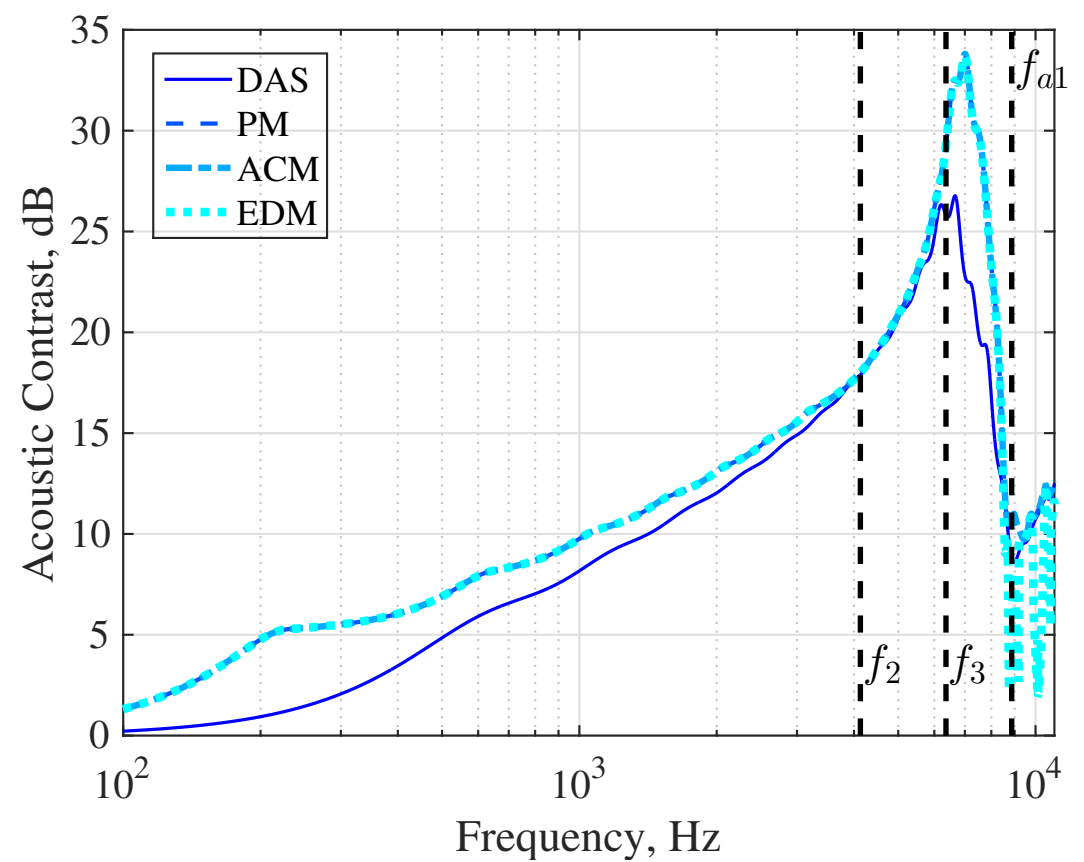

(a)

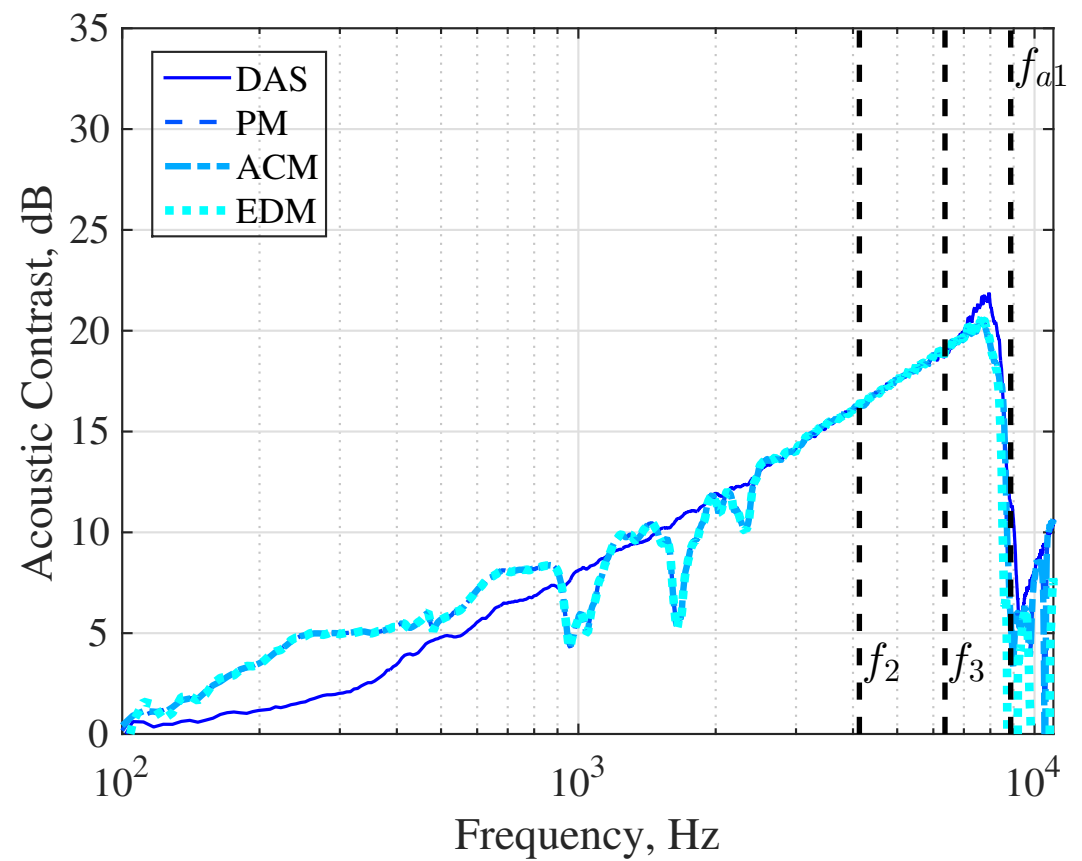

(b)

Figure 12: Simulated (a) and measured (b) acoustic contrast for the front beam due to filters designed with four beamforming methods. 
1 the same contrast. Above $f_{3}$ and up to the aliasing frequency, the optimal approaches provide higher contrast than the DAS. An interesting analysis is that of the measured acoustic contrast due to filters designed with $\mathbf{Z}$. In this case, the TFs used for the filter design and evaluation of performance are different, the latter being the TFs of the array in the anechoic chamber at the time of the measurement of the filters response. If that is the case, as reported by Elliott et al. [3], higher values of regularization are needed in order to increase the robustness of the system. 7he drops in the measured contrast in Fig. 12(b) at frequencies between $900 \mathrm{~Hz}$ and $1 \mathrm{kHz}$ and at around $1.8 \mathrm{kHz}$ can be explained by referring to the analysis of the mismatches between drivers performed in Section 5.1 (see Fig. 6(b)). This indicates that the values of the tunable parameters (the regularization, in particular) are not high enough to ensure system robustness. This, in turn, implies that the value of $E_{\mathrm{MAX}}$ was chosen to be too high for the implementation of the designed filters with the linear array prototype. A lower value of $E_{\mathrm{MAX}}$ in the frequency regions where the array is more sensitive to mismatches between drivers would have produced higher values of regularization parameter and lower values of tunable parameter $\alpha$.

The magnitude FRFs of the measured responses of the filters designed with DAS and PM at the bright point are shown in Fig. 13(a) together with the magnitude FRF of $\tilde{H}$. A real valued gain was applied to $\tilde{H}$ to allow for a better comparison with the measured responses. The measured responses of the filters designed by means of the beamforming methods and TF matrix $\mathbf{Z}$ (monopoles in free-field) resemble the response of the real loudspeakers. The differences at very low and very high frequencies between $\tilde{H}$ and the measured responses may be due to the effects of the Butterworth band-pass filter. The IRs of the measured responses of the filters and the impulse response of $\tilde{H}$ are shown in Fig. 13(b) and they provide an indication of the audio quality of the designed filters. We observe that the IRs of the measured responses have little pre-ringing but they show some post-ringing. This holds true for both DAS and PM. However, a thorough assessment of the audio quality of the filters requires subjective testing. A software was created that allows the reader to listen to the IRs of the measured responses of the filters at each control point. The software is available for download from the link provided in bibliographic reference [29]. 


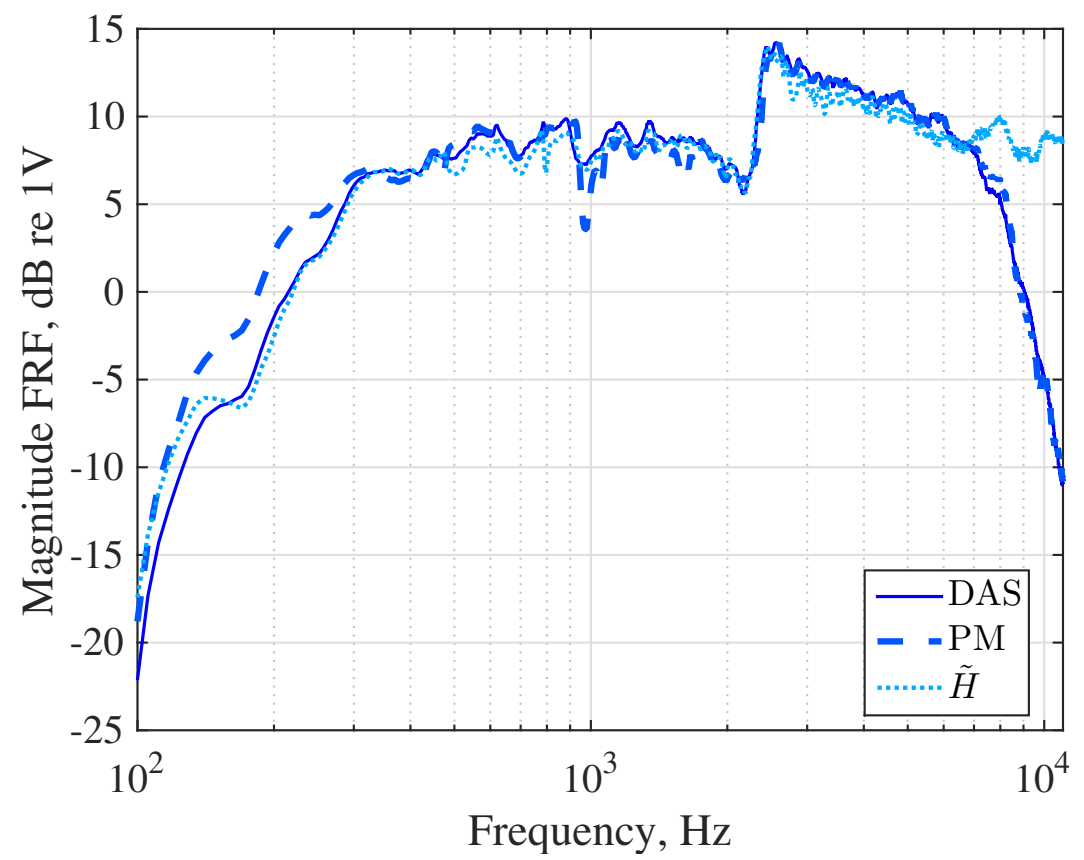

(a)

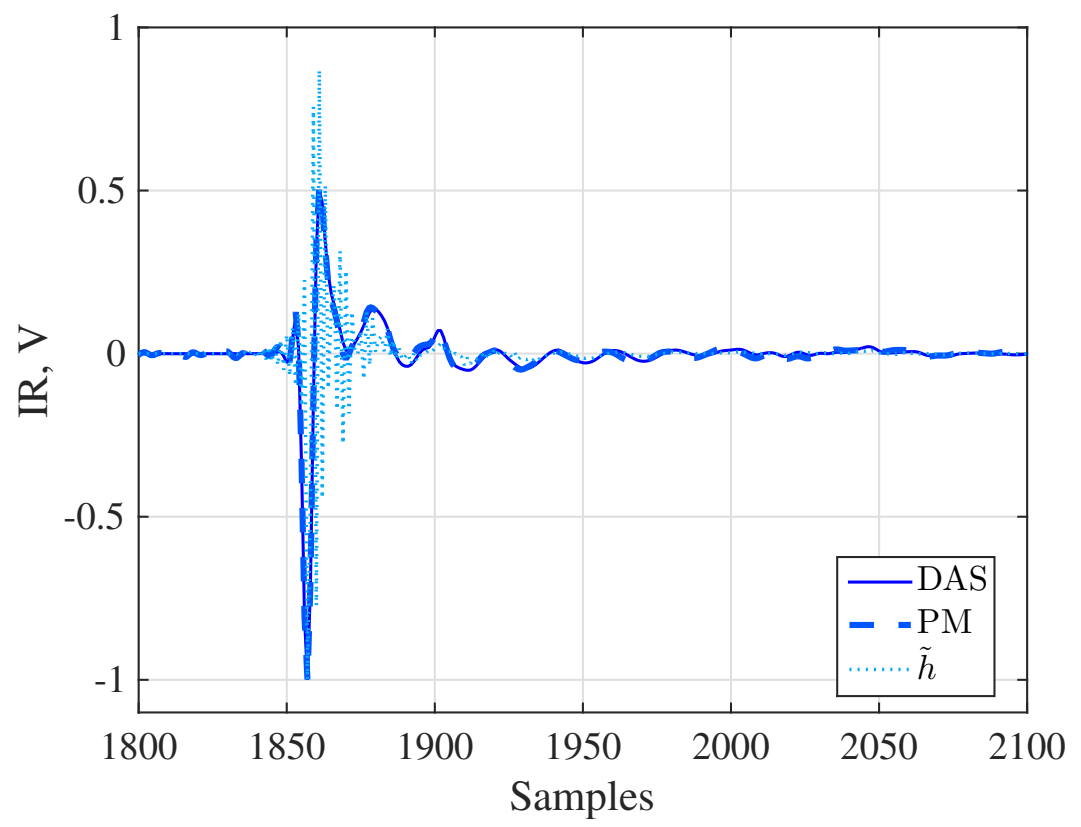

(b)

Figure 13: Front Beam. FRFs (a) and IRs (b) of the measured filters responses of the DAS and PM methods and $\tilde{H}$. 


\section{5.5. Discussion of results}

By imposing the performance constraints, performance of the optimal methods can be divided into three regions as a function of frequency, which are summarized in Fig. 14 and can be characterized as follows.

- From low frequencies up to $f_{1}$, the compensated filters designed with the optimal methods are identical between each other. Both the required input energy and the directivity performance are higher than that of the DAS. Above $f_{1}$, the tunable parameters of the optimal methods become equal to $\beta_{\mathrm{MIN}}$ (PM and $\mathrm{ACM}$ ) and $\alpha_{\mathrm{MAX}}$ (EDM).

- Above the transition band that goes from $f_{1}$ to $f_{2}$, in the second region (that goes from $f_{2}$ to $f_{3}$ ) the optimal methods perform like the DAS.

- The third region is at frequencies above the spatial aliasing limit $f_{\mathrm{a}}$. A prediction of the values of $f_{1}, f_{2}$, and $f_{3}$ is possible. We consider the case of the front beam and filters designed with the compensated PM method (due to the fact that we have shown that the ACM and EDM are identical below $f_{1}$ ). In order to estimate the value of $f_{1}$, we calculate the compensated filters $\hat{\mathbf{q}}_{\mathrm{PM}}$ designed with the PM method with no regularization applied (i.e., $\left.\hat{\mathbf{q}}_{\mathrm{PM}}(\beta=0)=W \mathbf{q}_{\mathrm{PM}}(\beta=0)\right)$, that is

$$
W \mathbf{q}_{\mathrm{PM}}(\beta=0)=\frac{\hat{p}_{B}}{\mathbf{z}_{B}^{T}\left(\mathbf{Z}^{H} \mathbf{Z}\right)^{-1} \mathbf{z}_{B}^{*}}\left(\mathbf{Z}^{H} \mathbf{Z}\right)^{-1} \mathbf{z}_{B}^{*},
$$

where we have used the relation $\mathbf{Z}^{H} \hat{\mathbf{p}}=\mathbf{z}_{B}^{*}$, which holds if the target signal is chosen as in Eq. (9). The frequency $f_{1}$ can be calculated by finding

$$
f_{1}=\min _{f}\left\{\left\|W(f) \mathbf{q}_{\mathrm{PM}}(f, \beta=0)\right\|^{2}=E_{\mathrm{MAX}}(f)\right\},
$$

where the operator $\min _{f}\{\cdot\}$ returns the smallest $f$ that satisfies the equation in $\{\cdot\}$. The value of $f$ that satisfies Eq. (32) is the frequency at which $\left\|\hat{\mathbf{q}}_{\mathrm{PM}}(\beta=0)\right\|^{2}$ intersects $E_{\mathrm{MAX}}$, as shown in Fig. 15(a), where the estimated frequency $f_{1}$ using Eq. (32) is equal to frequency $f_{1}$ shown in Fig. 7(a).

The frequencies $f_{2}$ and $f_{3}$ above which the optimal methods have the same input energy as the DAS up to the aliasing frequency depend instead on the TF matrix $\mathbf{Z}$. Let us consider the norm of $\hat{\mathbf{q}}_{\mathrm{PM}}(\beta=0)$, that is 


$$
\left\|\hat{\mathbf{q}}_{\mathrm{PM}}(\beta=0)\right\|=\left\|W\left(\mathbf{Z}^{H} \mathbf{Z}\right)^{-1} \mathbf{z}_{B}^{*}\right\| \leq\left\|W\left(\mathbf{Z}^{H} \mathbf{Z}\right)^{-1}\right\|\left\|\mathbf{z}_{B}^{*}\right\|=|W|\left\|\left(\mathbf{Z}^{H} \mathbf{Z}\right)^{-1}\right\|\left\|\mathbf{z}_{B}^{*}\right\|
$$

1 and the norm of the input signals designed with the DAS, that is

$$
\left\|\mathbf{q}_{\mathrm{DAS}}\right\|=\left\|\boldsymbol{\Gamma} \mathbf{z}_{B}^{*}\right\| \leq\|\boldsymbol{\Gamma}\|\left\|\mathbf{z}_{B}^{*}\right\|
$$

2 By direct comparison of Eq. (33) and Eq. (34), we see that if $|W|\left\|\left(\mathbf{Z}^{H} \mathbf{Z}\right)^{-1}\right\|=\|\boldsymbol{\Gamma}\|$, then $\left\|\hat{\mathbf{q}}_{\mathrm{PM}}\right\|$ 3 and $\left\|\mathbf{q}_{\text {DAS }}\right\|$ have the same upper bound to their norm. $\left\|\left(\mathbf{Z}^{H} \mathbf{Z}\right)^{-1}\right\|$ is defined as the largest 4 singular value of $\left(\mathbf{Z}^{H} \mathbf{Z}\right)^{-1}$. The values of $f_{2}$ and $f_{3}$ can be approximated as

$$
\begin{aligned}
& f_{2} \approx \min _{f}\left\{|W|\left\|\left(\mathbf{Z}^{H} \mathbf{Z}\right)^{-1}\right\| \approx\|\boldsymbol{\Gamma}\|\right\}, \\
& f_{3} \approx \max _{f}\left\{|W|\left\|\left(\mathbf{Z}^{H} \mathbf{Z}\right)^{-1}\right\| \approx\|\boldsymbol{\Gamma}\|\right\},
\end{aligned}
$$

respectively, where $\max _{f}\{\cdot\}$ returns the largest $f$ that satisfies the equation in $\{\cdot\}$. The frequencies $6 \quad f_{2}$ and $f_{3}$ are, respectively, the first and last frequencies at which $|W|\left\|\left(\mathbf{Z}^{H} \mathbf{Z}\right)^{-1}\right\|$ is almost equal 7 to $\|\boldsymbol{\Gamma}\|$ as shown in Fig. 15(b). Interestingly, this suggests that $\mathbf{Z}^{H} \mathbf{Z}$ becomes increasingly 8 diagonally dominant with increasing frequency. This, in turn, suggests that, in the frequency range 9 where $|W|\left\|\left(\mathbf{Z}^{H} \mathbf{Z}\right)^{-1}\right\| \approx\|\boldsymbol{\Gamma}\|$, the columns of $\mathbf{Z}^{H} \mathbf{Z}$ are orthogonal. If we are in the far-field, the estimation of the two frequencies simplifies further, because the diagonal matrix $\Gamma$ can be approximated as $\Gamma \approx \hat{\gamma} \mathbf{I}$, where $\hat{\gamma}=\left(4 \pi R^{2}\right) / L=E_{\mathrm{REF}}$ and $E_{\mathrm{REF}}$ is defined in Eq. (22). If that is the case, then

$$
\left\|\mathbf{q}_{\mathrm{DAS}}\right\| \approx \hat{\gamma}\left\|\mathbf{z}_{B}^{*}\right\|
$$

13 and the values of $f_{2}$ and $f_{3}$ can be approximated as $f_{2} \approx \min _{f}\left\{|W|\left\|\left(\mathbf{Z}^{H} \mathbf{Z}\right)^{-1}\right\| \approx \hat{\gamma}\right\}$ and $f_{3} \approx$ ${ }_{14} \max _{f}\left\{|W|\left\|\left(\mathbf{Z}^{H} \mathbf{Z}\right)^{-1}\right\| \approx \hat{\gamma}\right\}$, respectively.

15

To summarize, the value of $f_{1}$ depends on the TF matrix and the value of $E_{\mathrm{MAX}}$. It is important because below $f_{1}$, the regularization parameter is higher than $\beta_{\mathrm{MIN}}$. For a given TF matrix, the higher the value of $E_{\mathrm{MAX}}$, the lower the $f_{1}$. Instead, the frequencies $f_{2}$ and $f_{3}$ depend on the TF only. 


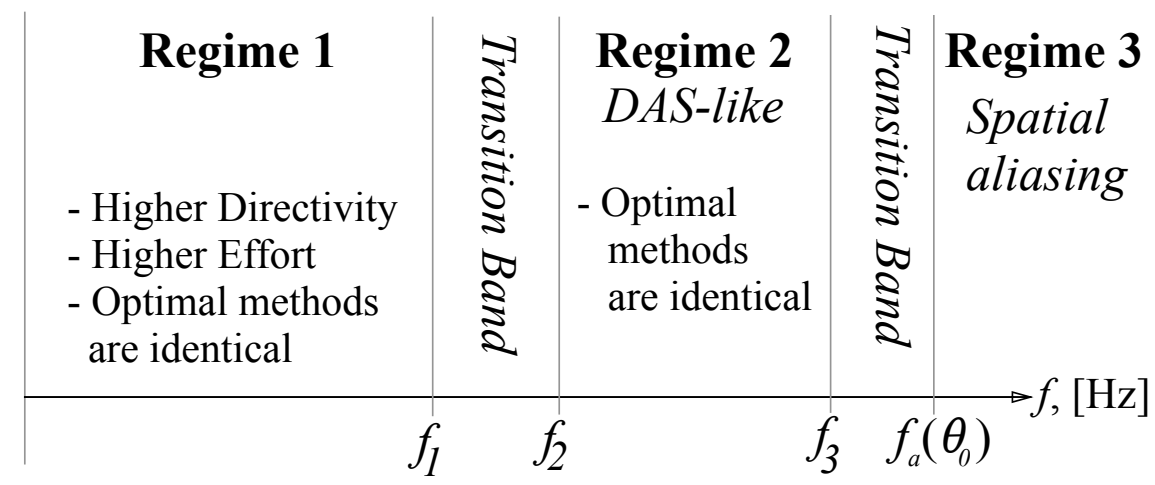

Figure 14: Summary of the three regions.

\subsection{Results for the side beam}

With the aim of verifying whether the results obtained for the front beam can be generalized, the same analysis is performed for the beam steered to the side of the array. In this case, the filters

4 for the side beam were designed with the algorithms described in Section 4.2 using the same procedure as the one adopted for the front beam. Fig. 16(a) shows that the input energy of the filters designed for the side beam meets the constraint on the limit on the maximum input energy. In this case, for filters designed with the PM and ACM methods, a frequency $f_{4} \approx 3.5 \mathrm{kHz}>f_{1}$ exists at which the input energy drops. This frequency is calculated by means of the method presented in the previous section and Eq. (32). Fig. 16(b) shows the magnitude of the FRFs of the optimal filters for loudspeaker \# 5 for the side beam. Below $f_{4}$ filters are all identical, whilst PM and ACM are identical across the whole frequency range. The frequency range where the filters designed with optimal beamforming methods resemble that of the DAS is $f_{5}<f<f_{6}$, where $f_{5}$ 3 and $f_{6}$ were calculated with Eq. (35) and Eq. (36), respectively. This range is narrower than that observed for the front beam.

In general, the same trends in performance as for the front beam are also noticed in the results for the side beam. The subplots in Fig. 17 and Fig. 18 show the simulated and measured array

efficiency and acoustic contrast for the side beam, respectively. In terms of acoustic contrast, there

is better agreement between simulated and measured results. The effects of the regularization and driver mismatches on the robustness of the system can be noticed also in this case. 


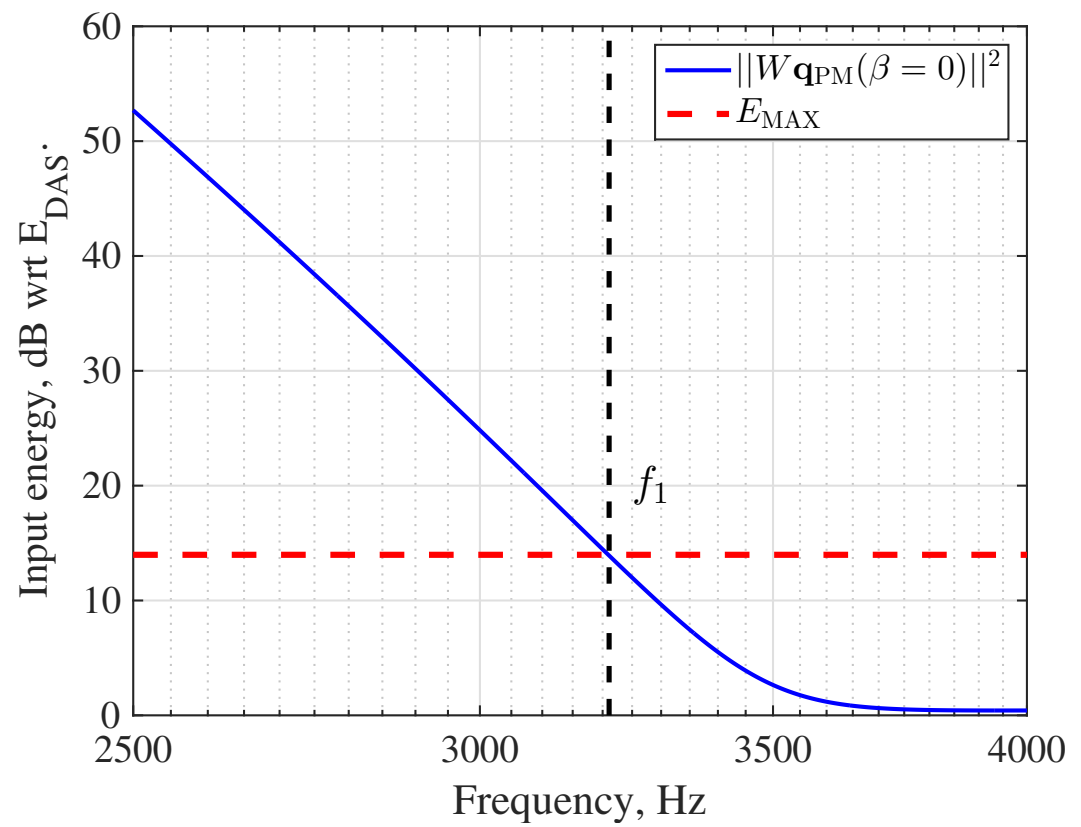

(a)

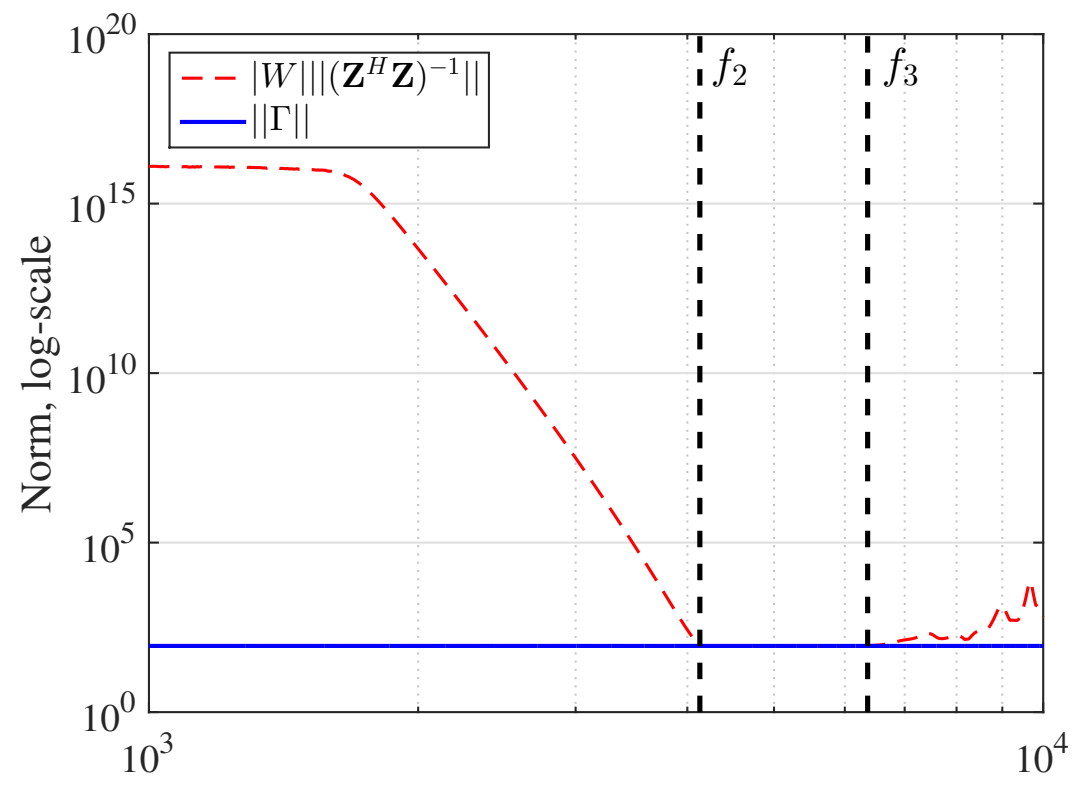

Frequency, $\mathrm{Hz}$

(b)

Figure 15: Estimation of frequency $f_{1}(\mathrm{a}), f_{2}$ and $f_{3}$ (b) for the front beam. 


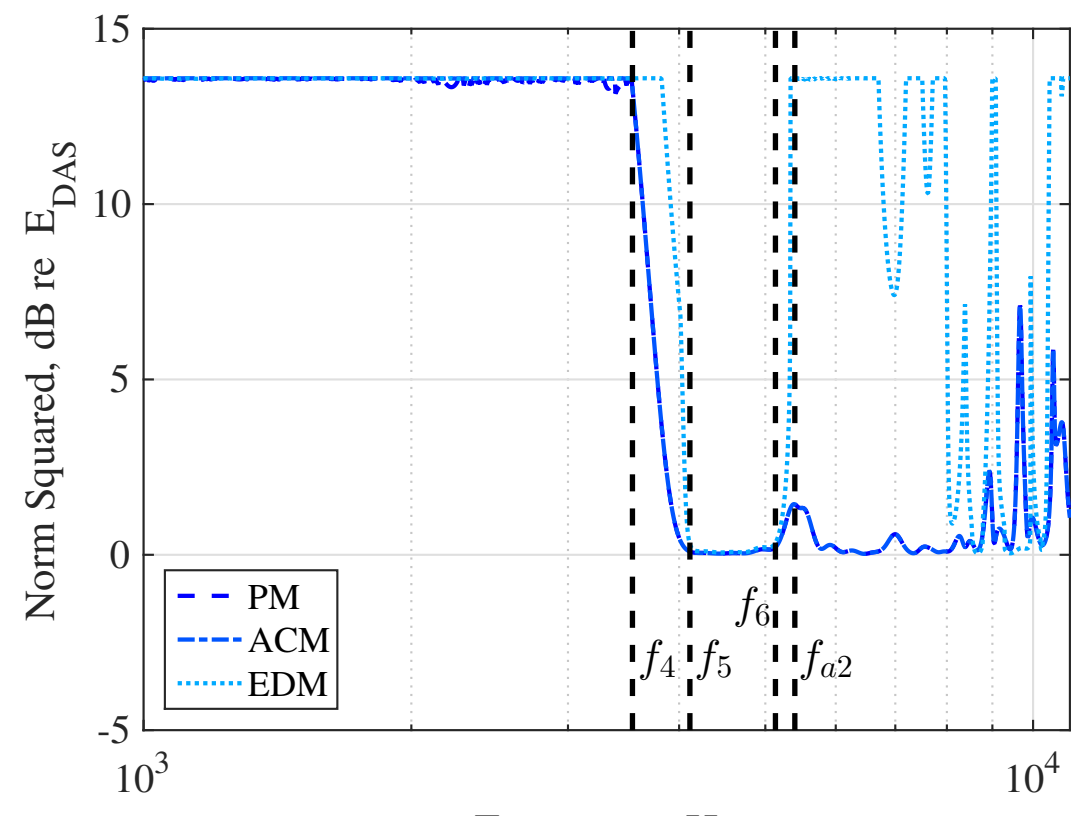

Frequency, $\mathrm{Hz}$

(a)

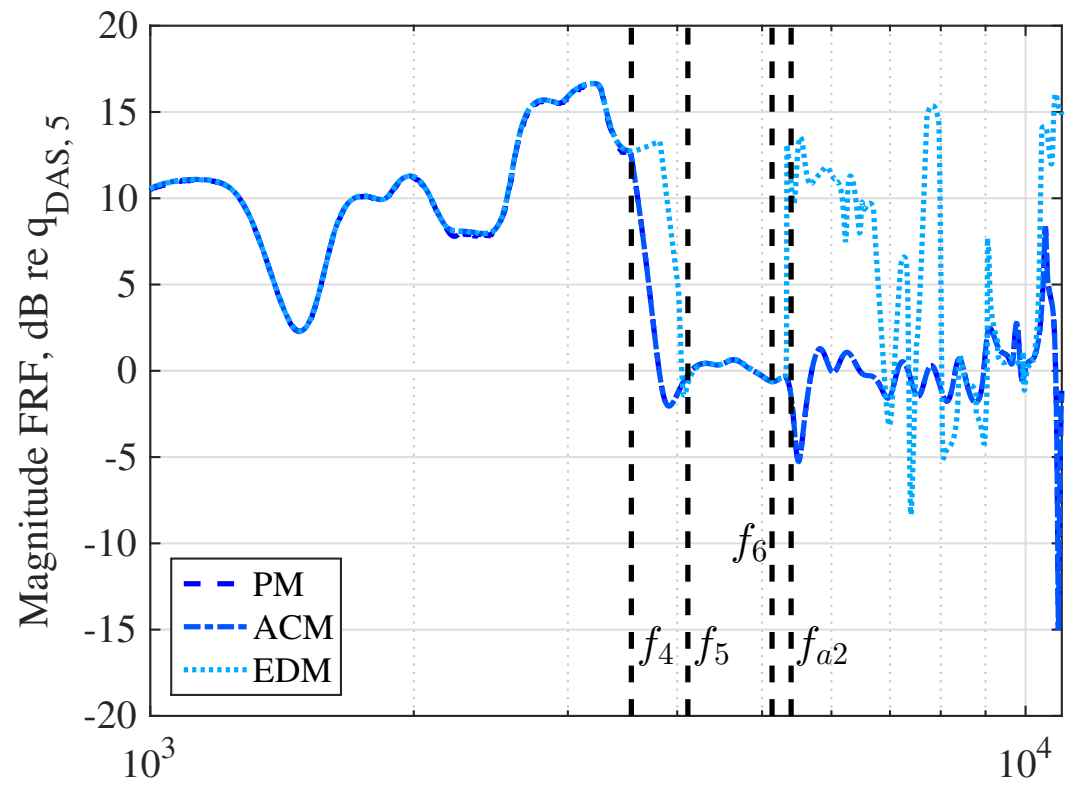

Frequency, $\mathrm{Hz}$

(b)

Figure 16: Side beam. Input energy required by the designed filters (a). Magnitude FRF of the optimal filters (PM, ACM, EDM) for loudspeaker \#5 normalized by the response of the DAS filter for loudspeaker \#5 (b). The vertical dashed lines are the frequencies indicating the three regions for the side beam. 


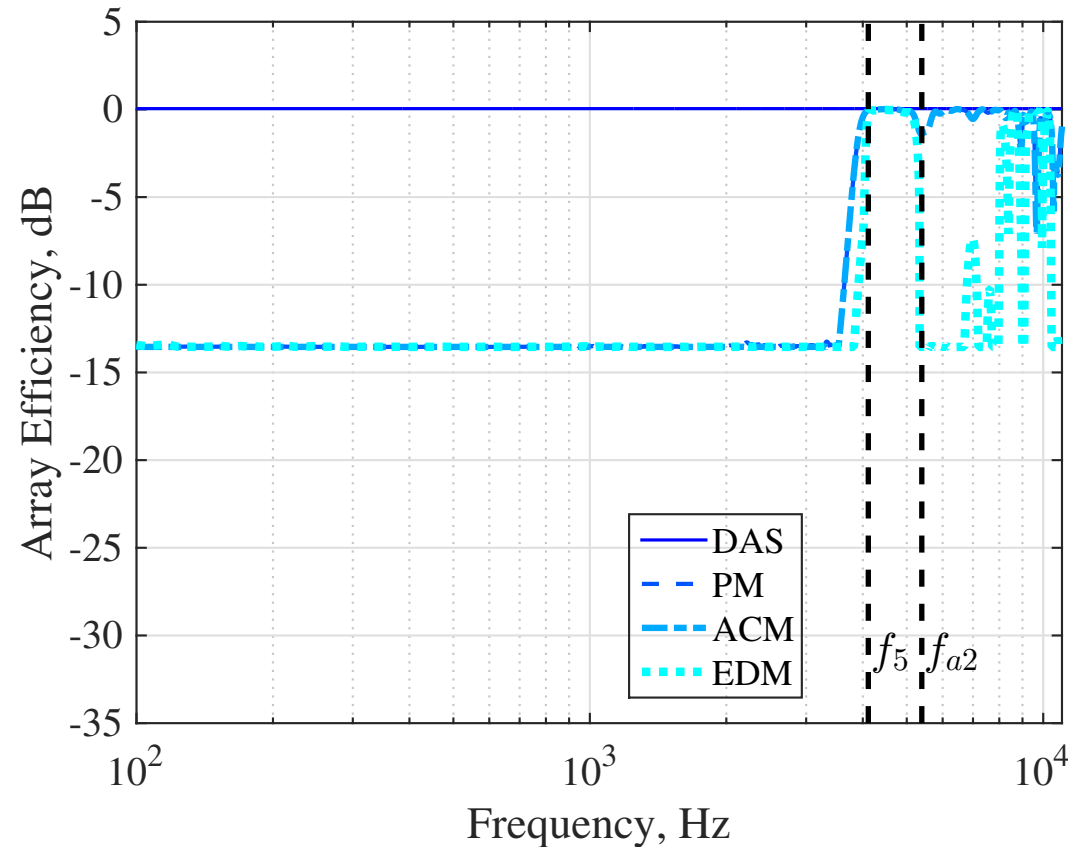

(a)

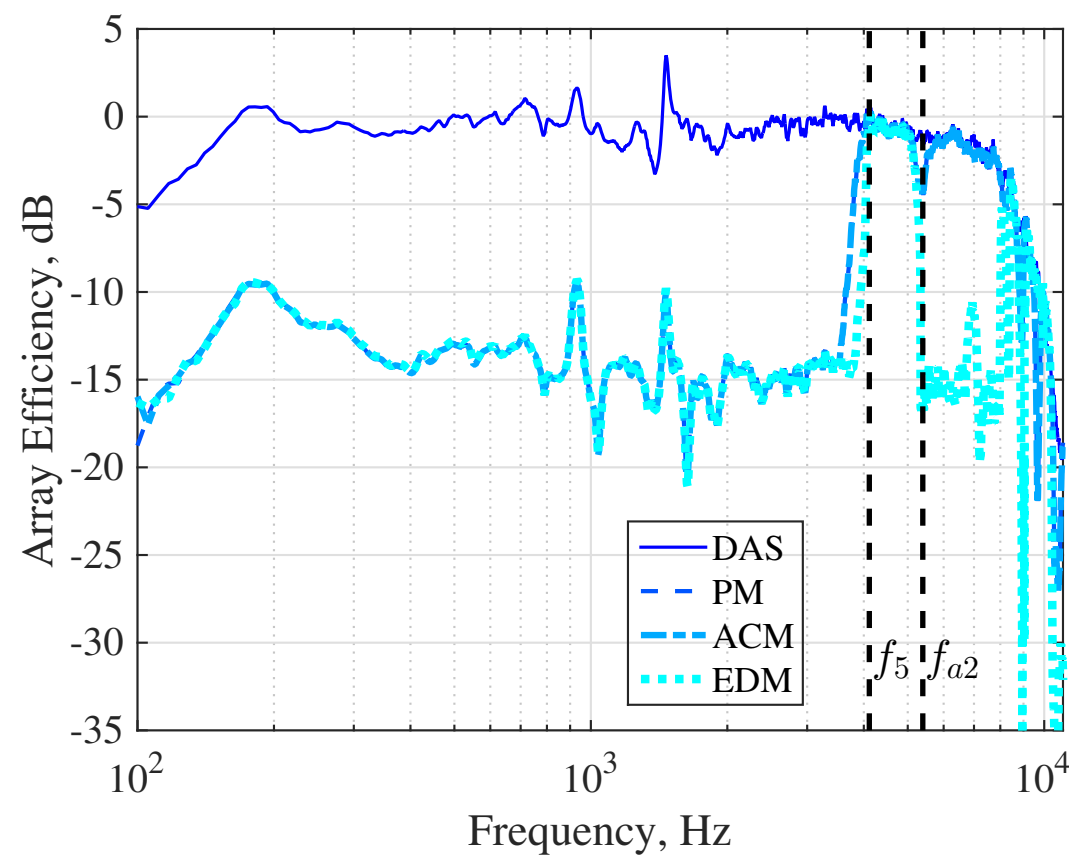

(b)

Figure 17: Simulated (a) and measured (b) array efficiency for the side beam due to filters designed with four beamforming methods. 


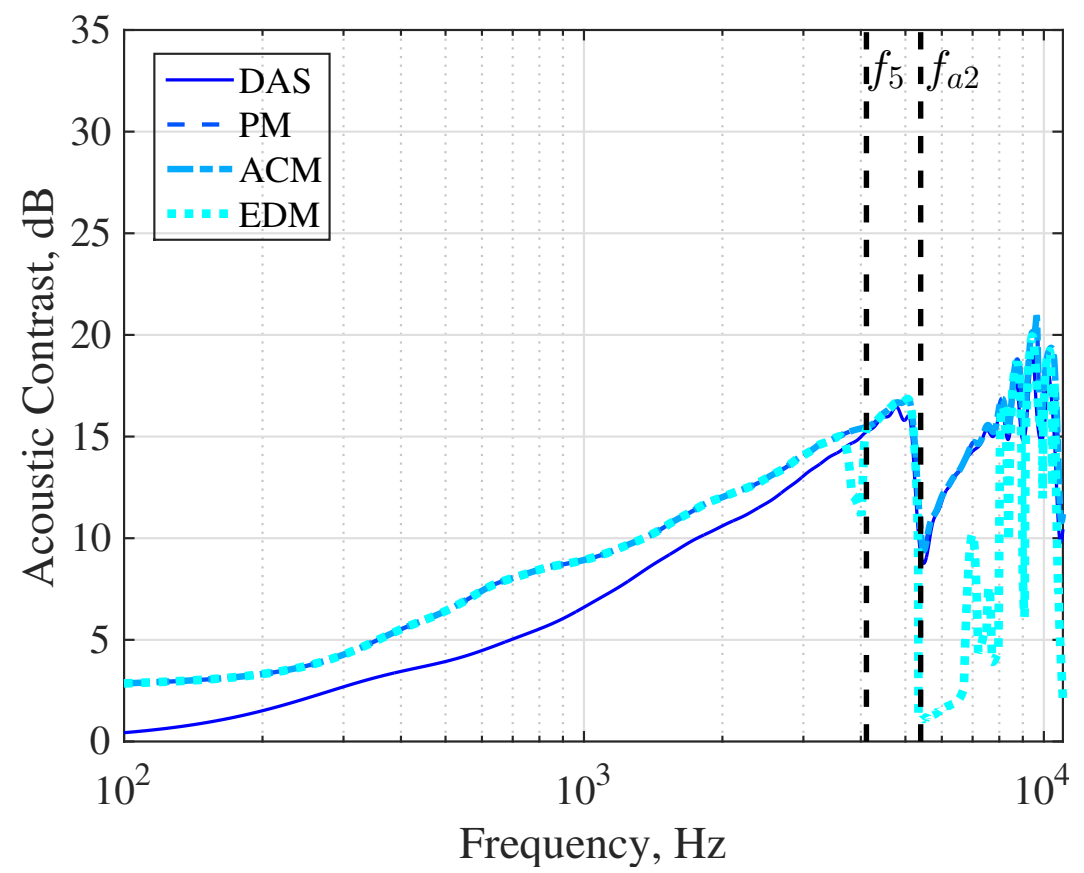

(a)

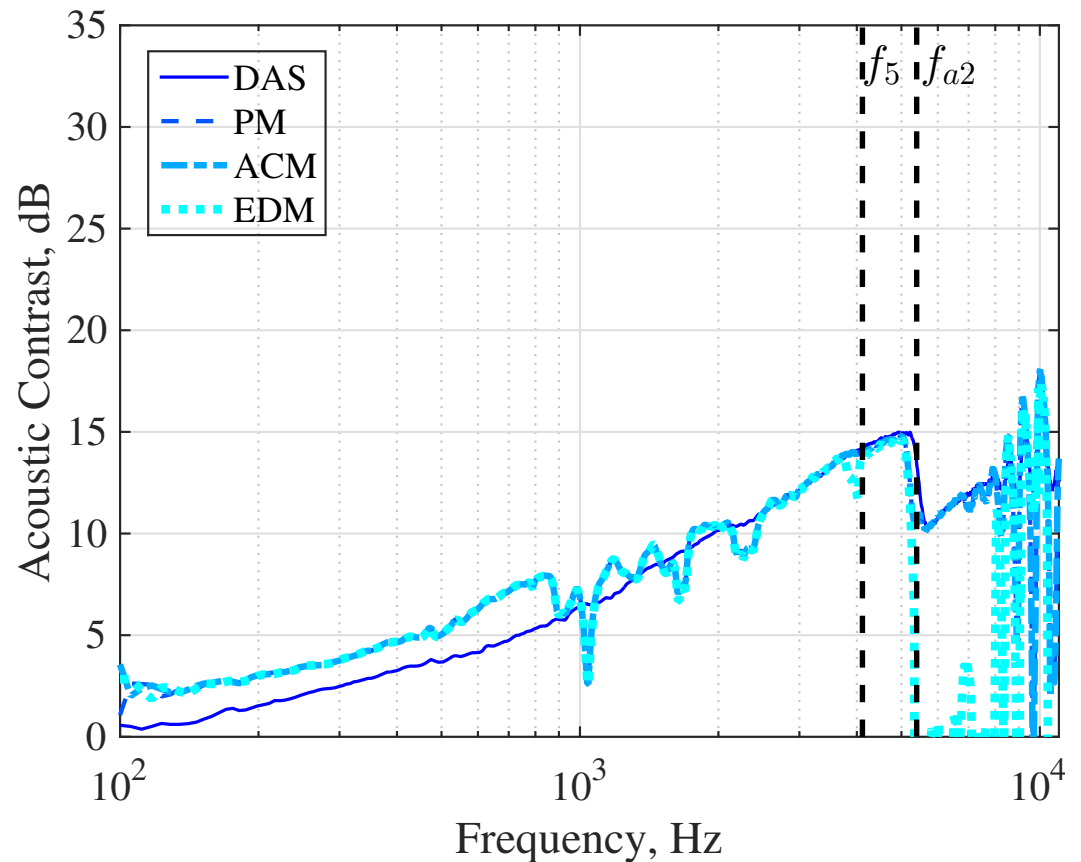

(b)

Figure 18: Simulated (a) and measured (b) acoustic contrast for the side beam due to filters designed with four beamforming methods. 


\section{Conclusions}

In this work, a framework for the comparison of beamforming methods for loudspeaker arrays in personalized audio applications has been presented and validated by means of numerical simulations and experiments under free-field conditions using a linear array prototype. More specifically, the delay-and-sum and three strategies for optimal beamforming (i.e., Pressure Matching, Acoustic Contrast Maximization, and Energy Difference Maximization methods) were compared under given performance constraints on the maximum input energy to the array and frequency response function of the sound field reproduced by the array at the listener's position, which relate to the limited power handling capabilities of compact arrays and the characteristics of the sound field delivered to the listener, respectively. Filters were designed using a monopole source model for sound radiation. Where applicable, the tunable parameters of each method and a filter for compensation of the response of the sound field at the listener's position were calculated in order to meet the performance constraints. Two beamforming problems were considered for the linear loudspeaker array, i.e. beams steered to the front and side of the array, respectively.

For both beams and under the given performance constraints, results of the simulations and experiments are characterized by three regions in the frequency domain. In the first region, at low frequencies and whose limit depends on the limit to the input energy, input signals designed with the optimal methods are identical between each other and provide higher directivity than that of the delay-and-sum at the expense of required higher input energy at low frequencies. The second region exists in the mid-frequency range, where the optimal methods perform like the delay-and-sum. At high frequencies, above the spatial aliasing limit, performance of Pressure Matching and Acoustic Contrast Maximization are identical. Instead, that of the Energy Difference Maximization largely depend on the choice of the tunable parameter. An estimation method for the frequency boundaries of the three regions in the case of the Pressure-Matching method has been presented.

In the case of the designed filters implemented with a real array, the results of the experiments in anechoic environment have shown the effects of the tunable parameters on the robustness of the system, which are particularly noticeable in the mid-frequency range due to the mismatch between the transducers of the considered linear array prototype. 


\section{Acknowledgments}

2 This work has been partially funded by Huawei and by the Royal Academy of Engineering 3 and by the Engineering and Physical Sciences Research Council, UK. The authors would like

4 to express their gratitude to Peter Otto for his contribution on the realization of the linear array 5 prototype and to Phil Oxborrow for his contribution during the experimental session.

\section{A. Proof of PM compensated solution}

$7 \quad$ By considering the PM problem in Eq. (6), we seek for solutions of the form $\hat{\mathbf{q}}=C \mathbf{q}$, where $8 \quad C \in \mathbb{C}, C \neq 0$, that is

$$
\begin{array}{r}
J_{\mathrm{PM}}(\hat{\mathbf{q}})=(\hat{\mathbf{p}}-C \mathbf{Z} \mathbf{q})^{H}(\hat{\mathbf{p}}-C \mathbf{Z q})+\beta_{\mathrm{PM}} C^{*} C \mathbf{q}^{H} \mathbf{q} \\
=\hat{\mathbf{p}}^{H} \hat{\mathbf{p}}-C \hat{\mathbf{p}}^{H} \mathbf{Z q}-C^{*} \mathbf{q}^{H} \mathbf{Z}^{H} \hat{\mathbf{p}}+\left(C^{*} C\right) \mathbf{q}^{H} \mathbf{Z}^{H} \mathbf{Z q}+\left(C^{*} C\right) \beta_{\mathrm{PM}} \mathbf{q}^{H} \mathbf{q} .
\end{array}
$$

9 The partial derivative of $J_{\mathrm{PM}}(\hat{\mathbf{q}})$ with respect to $\mathbf{q}$ is

$$
\begin{array}{r}
\frac{\partial J_{\mathrm{PM}}(\hat{\mathbf{q}})}{\partial \mathbf{q}}=\frac{\partial \hat{\mathbf{p}}^{H} \hat{\mathbf{p}}}{\partial \mathbf{q}}-\frac{\partial C \hat{\mathbf{p}}^{H} \mathbf{Z q}}{\partial \mathbf{q}}-\frac{\partial C^{*} \mathbf{q}^{H} \mathbf{Z}^{H} \hat{\mathbf{p}}}{\partial \mathbf{q}} \\
+\frac{\partial\left(C^{*} C\right) \mathbf{q}^{H} \mathbf{Z}^{H} \mathbf{Z} \mathbf{q}}{\partial \mathbf{q}}+\frac{\partial\left(C^{*} C\right) \beta_{\mathrm{PM}} \mathbf{q}^{H} \mathbf{q}}{\partial \mathbf{q}} .
\end{array}
$$

By setting Eq. (39) to zero, that is

$$
\begin{array}{r}
\frac{\partial J_{\mathrm{PM}}(\hat{\mathbf{q}})}{\partial \mathbf{q}}=- \\
-2 C^{*} \mathbf{Z}^{H} \hat{\mathbf{p}}+2\left(C^{*} C\right)\left(\mathbf{Z}^{H} \mathbf{Z}+\beta_{\mathrm{PM}} \mathbf{I}\right) \mathbf{q}= \\
2 C^{*}\left[-\mathbf{Z}^{H} \hat{\mathbf{p}}+C\left(\mathbf{Z}^{H} \mathbf{Z}+\beta_{\mathrm{PM}} \mathbf{I}\right) \mathbf{q}\right]=0,
\end{array}
$$

11 and by solving the above equation with respect to $\mathbf{q}$, we obtain the solution to the modified PM

12 problem, that is

$$
\hat{\mathbf{q}}=\frac{1}{C}\left(\mathbf{Z}^{H} \mathbf{Z}+\beta_{\mathrm{PM}} \mathbf{I}\right)^{-1} \mathbf{Z}^{H} \hat{\mathbf{p}} .
$$


1 This can be expressed as a function of $\mathbf{q}_{\mathrm{PM}}$ as

$$
\hat{\mathbf{q}}=\frac{1}{C} \mathbf{q}_{\mathrm{PM}}
$$

2 This proves that the solution $\hat{\mathbf{q}}$ is related to the solution of the PM problem in Eq. (6). We now find an analytical expression for $C$ by applying the constraint on the reproduced pressure at the chosen bright point, that is

$$
\mathbf{z}_{B}^{T} \hat{\mathbf{q}}=\hat{p}_{B}
$$

5 By substituting Eq. (42) into Eq. (43) and solving with respect to $C$, we can write

$$
C=\frac{\mathbf{z}_{B}^{T} \mathbf{q}_{\mathrm{PM}}}{\hat{p}_{B}}
$$

6 Substituting the above equation back into Eq. (42), we obtain

$$
\hat{\mathbf{q}}=\frac{\hat{p}_{B}}{\mathbf{z}_{B}^{T} \mathbf{q}_{\mathrm{PM}}} \mathbf{q}_{\mathrm{PM}},
$$

7 that is the expression of the compensated filters that we have used to implement the constraints.

\section{B. Procedure for the calculation of $\alpha_{\mathrm{MAX}}$}

Shin et al. [8] have reported that the ability of loudspeaker array driven with signals designed with the EDM method to provide a high energy difference between bright and dark zone highly depends on the geometry of the array and control point arrangement, especially at low frequencies. More specifically, higher values of energy difference (higher values of $\alpha$ ) may result in systems that do not provide the expected performance. This aspect is further investigated in this work and used for the calculation of $\alpha_{\mathrm{MAX}}$. For a given set of filter $\tilde{\mathbf{q}}_{\mathrm{EDM}}$ and a given frequency, the idea is to calculate $\alpha_{\mathrm{MAX}}$ as the maximum value of $\alpha$ before the system becomes unstable.

With reference to the beamforming problem in Fig. 4(a), we calculate $\tilde{\mathbf{q}}_{\text {EDM }}$ using Eq. (18) and Eq. (17) with no constraint on the input energy. The tunable parameters $\alpha$ varies between $0<\alpha<\tilde{\alpha}$, where $\tilde{\alpha}$ is set to $\tilde{\alpha}=200$ for the purpose of this simulations. As shown in Fig. 19(a), the acoustic contrast at $125 \mathrm{~Hz}$ increases almost regularly as a function of $\alpha$ up to $\alpha \approx 10$. For 
$1 \quad \alpha>10$, the system becomes unstable. Similarly, at $2 \mathrm{kHz}$, the systems becomes unstable above $2 \alpha \approx 30$. At $4 \mathrm{kHz}$ the $A C$ is constant as a function of $\alpha$ and this indicates that $4 \mathrm{kHz}$ belongs to 3 the frequency range where the system is able to provide a larger energy difference between sound 4 zones. These considerations are used for the calculation of the value of $\alpha_{\mathrm{MAX}}$. The assumption is made that the $A C$ increases regularly and almost linearly up to the value of $\alpha=\alpha_{\mathrm{MAX}}$. If the $A C$ increases linearly, the derivative of $A C$ with respect to $\alpha$ is constant. Thus, the second derivative of the $A C$ with respect to $\alpha$, should be equal to zero up to $\alpha=\alpha_{\mathrm{MAX}}$ and erratic for $\alpha>\alpha_{\mathrm{MAX}}$.

8 The value of $\alpha_{\mathrm{MAX}}$ is selected as the value of $\alpha$ for which the second derivative of the $A C$ does 9 not exceed a small threshold $\delta_{\text {EDM }}$ set by the user, that is

$$
\alpha_{\mathrm{MAX}}=\alpha:-\delta_{\mathrm{EDM}}<\frac{\partial^{2} A C(\alpha)}{\partial \alpha^{2}}<\delta_{\mathrm{EDM}}
$$

where $\delta_{\mathrm{EDM}}$ has been set as $\delta_{\mathrm{EDM}}=10^{-2}$. The second partial derivative of the $A C$ as a function of $\alpha$ at $125 \mathrm{~Hz}$ is shown in Fig. 19(b) is almost equal to zero up to the value of $\alpha \approx 10$, which is the same as the one found in Fig. 19(a). Similar considerations hold for the second partial derivative of $A C$ with respect to $\alpha$ at $2000 \mathrm{~Hz}$ as shown in Fig. 19(c). The values of $\alpha_{\text {MAX }}$ (as a function of frequency) calculated with the procedure described above are shown in Fig. 19(d).

\section{References}

[1] F. Olivieri, F. M. Fazi, M. Shin, P. Nelson, S. Fontana, Y. Lang, Numerical comparison of sound field control strategies under free-field conditions for given performance constraints. In Institute of Acoustics - Reproduced Sound 2014 (Vol. 1). Birmingham, UK, 2014

[2] W. Druyvesteyn, J. Garas, Personal sound, Journal of the Audio Engineering Society 45 (9) (1997) 685-701.

[3] S. J. Elliott, J. Cheer, J. W. Choi, Y. Kim, Robustness and Regularization of Personal Audio Systems, IEEE Transactions on Audio, Speech, and Language Processing 20 (7) (2012) 2123-2133. doi:10.1109/TASL.2012.2197613.

[4] M. Fink, C. Prada, Acoustic time-reversal mirrors, (2001), Inverse Problems, 17(1), R1R38. doi:10.1088/0266$5611 / 17 / 1 / 201$

[5] O. Kirkeby, P. A. Nelson, Reproduction of plane wave sound fields, The Journal of the Acoustical Society of America 94 (1993) 2992. doi:10.1121/1.407330.

[6] J. W. Choi, Y. H. Kim, Generation of an acoustically bright zone with an illuminated region using multiple sources, The Journal of the Acoustical Society of America 111 (4) (2002) 1695. doi:10.1121/1.1456926. 


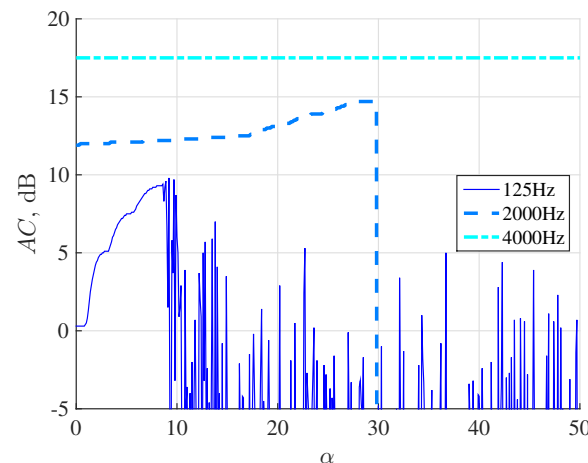

(a)

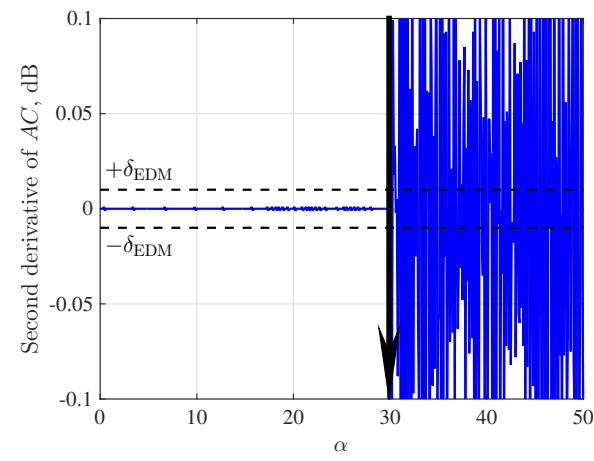

(c)

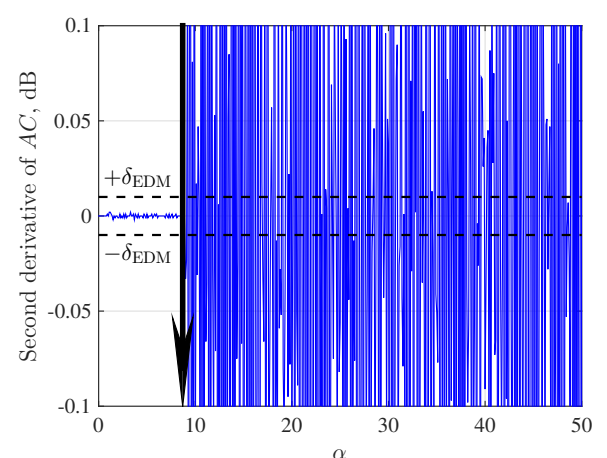

(b)

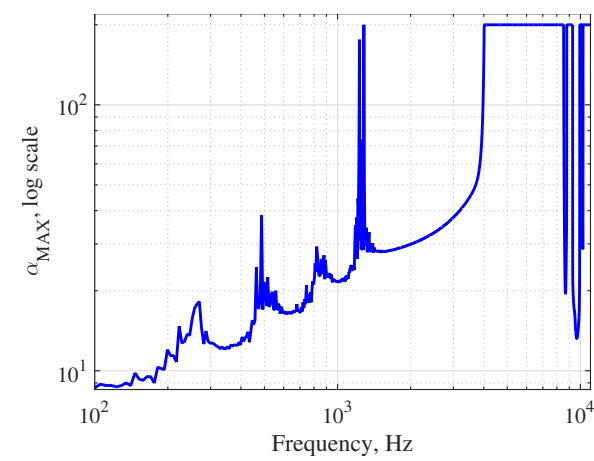

(d)

Figure 19: Procedure for the calculation of $\alpha_{\mathrm{MAX}}$. Acoustic contrast as a function of $\alpha$ (a). Second partial derivative of the acoustic contrast with respect to $\alpha$ at $125 \mathrm{~Hz}$ (b) and $2 \mathrm{kHz}$ (c), respectively, and selected values of $\alpha_{\text {MAX }}$ (indicated by the arrows). Selected $\alpha_{\operatorname{MAX}}$ as a function of frequency (d) using the procedure described in Section 4.2.

[7] M. Shin, S. Q. Lee, F. M. Fazi, P. A. Nelson, D. Kim, S. Wang, K. H. Park, J. Seo, Maximization of acoustic energy difference between two spaces, The Journal of the Acoustical Society of America 128 (1) (2010) 121-31. doi:10.1121/1.3438479.

[8] M. Shin, F. M. Fazi, P. A. Nelson, F. C. Hirono, Controlled sound field with a dual layer loudspeaker array, Journal of Sound and Vibration 333 (16) (2014) 3794-3817. doi:10.1016/j.jsv.2014.03.025.

[9] J. H. Chang, F. Jacobsen, Sound field control with a circular double-layer array of loudspeakers, The Journal of the Acoustical Society of America 131 (6) (2012) 4518. doi:10.1121/1.4714349.

[10] M. B. Mø ller, M. Olsen, F. Jacobsen, A Hybrid Method Combining Synthesis of a Sound Field and Control of Acoustic Contrast, in 132nd Audio Engineering Society Convention, Budapest, Hungary, 2006.

[11] Y. Cai, M. Wu, J. Yang, Sound reproduction in personal audio systems using the least-squares approach with 
acoustic contrast control constraint, The Journal of the Acoustical Society of America 135 (2) (2014) 734-741. doi:10.1121/1.4861341.

[12] J. Cheer, S. J. Elliott, A Comparison of Control Strategies for a Car Cabin Personal Audio System, inAudio Engineering Society 52nd International Conference: Sound Field Control - Engineering and Perception, Guildford, UK, 2013.

[13] A. Berkhoff, R. V. D. Rots, Directional sound sources using real-time beamforming control, in: 42nd Int. Congr. Expo. Noise Control Eng. - Noise Control Qual. Life - INTER-NOISE 2013, Innsbruck, Austria, 2013.

[14] M. Olik, J. Francombe, P. Coleman, P. J. B. Jackson, M. Olsen, M. Moller, S. Bech, A Comparative Performance Study of Sound Zoning Methods in a Reflective Environment, in Audio Engineering Society 52nd International Conference: Sound Field Control - Engineering and Perception, Guildford, UK, 2013.

[15] M. F. Simón Gálvez, S. J. Elliott, J. Cheer, A superdirective array of phase shift sources, The Journal of the Acoustical Society of America 132 (2) (2012) 746-56. doi:10.1121/1.4733556.

[16] P. Coleman, P. J. Jackson, M. Olik, M. Olsen, M. Møller, J. Abildgaard Pedersen, The influence of regularization on anechoic performance and robustness of sound zone methods, Proceedings of Meetings on Acoustics, Vol. 19, 2013. doi:10.1121/1.4799031.

[17] P. Coleman, P. J. B. Jackson, M. Olik, M. Møller, M. Olsen, J. Abildgaard Pedersen, Acoustic contrast, planarity and robustness of sound zone methods using a circular loudspeaker array, The Journal of the Acoustical Society of America 135 (June) (2014) 1929-1940. doi:10.1121/1.4866442.

[18] O. Kirkeby, P. Nelson, H. Hamada, F. Orduna-Bustamante, Fast deconvolution of multichannel systems using regularization, IEEE Transactions on Audio, Speech, and Language Processing 6 (2) (1998) $189-194$. doi:10.1109/89.661479.

[19] P. A. Nelson, S. J. Elliott, Active control of sound, Academic Press, 1993.

[20] O. Kirkeby, P. A. Nelson, F. Orduna Bustamante, H. Hamada, Local sound field reproduction using digital signal processing, The Journal of the Acoustical Society of America 100 (3) (1996) 1584-1593. doi:10.1121/1.416060.

[21] S. Spors, R. Rabenstein, Spatial Aliasing Artifacts Produced by Linear and Circular Loudspeaker Arrays used for Wave Field Synthesis, in 120th Audio Engineering Society Convention, Paris, France, 2006.

[22] ISVR, ISVR Anechoic Chamber Web site. URL http: / www. isvr. co. uk/faciliti/lg_anech. htm (Last visited January 2016)

[23] K. Shin, J. Hammond, Fundamentals of signal processing for sound and vibration engineers, Wiley, 2008.

[24] A. V. Oppenheim, R. W. Schafer, J. R. Buck, Discrete-time Signal Processing (2Nd Ed.), Prentice-Hall, Inc., Upper Saddle River, NJ, USA, 1999.

[25] M. R. Bai, Y.-H. Hsieh, Point focusing using loudspeaker arrays from the perspective of optimal beamforming, The Journal of the Acoustical Society of America 137(6) (2015), 33933410. doi:10.1121/1.4921602. 
[26] B. Masiero, M. Vorlander, A Framework for the Calculation of Dynamic Crosstalk Cancellation Filters. IEEE/ACM Transactions on Audio, Speech, and Language Processing, 22(9) (2014), 13451354. doi:10.1109/TASLP.2014.2329184.

4 [27] Y. Kim, P. A. Nelson. Optimal regularisation for acoustic source reconstruction by inverse methods. Journal of Sound and Vibration, 275(3-5) (2004), 463487. doi:10.1016/j.jsv.2003.06.031.

6 [28] T. Betlehem, W. Zhang, M. A. Poletti, T. D. Abhayapala. Personal Sound Zones: Delivering interface-free audio to multiple listeners. IEEE Signal Processing Magazine, 32(2) (2015), 8191. doi:10.1109/MSP.2014.2360707.

8 [29] F. Olivieri. Comparative analysis of beamforming methods - Audio demonstration (software download). URL https://zenodo.org/record/45638\#.VrZe5zaLSRs (Last visited January 2016). doi:10.5281/zenodo.45645. 


\section{List of Figure Captions}

1 (Color online) Example of a beamforming problem for personalized audio applications. A loudspeaker array is driven by purposefully designed input signals to focus sound where User 2 is located while minimizing sound pressure where User 1 and User 3 are located. . . . . . . . . . . . . . . . 6

2 Pseudo-code of the algorithms used for the calculation of the compensated filters for the PM and ACM

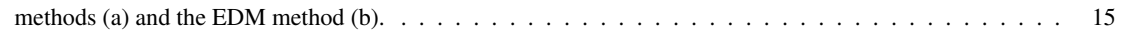

3 (Color online) The 16-channel linear loudspeaker array prototype and the loudspeaker numbering. . . . . 16

4 Beamforming problems for the linear array. Configurations of the front (a) and side (b) beams. . . . . . . 17

5 (Color online) The linear loudspeaker array prototype and microphone installed in the ISVR anechoic chamber (a) and block diagram of the experimental set-up (b) . . . . . . . . . . . . . . . . . . 21

6 Electro-acoustical analysis of the linear loudspeaker array. Magnitude FRF of the measured transfer functions between loudspeaker \#8 and two microphone positions (a). Standard deviation of measured and analytical responses at two microphone positions $(b) \ldots \ldots \ldots \ldots \ldots \ldots \ldots$

7 Front beam. Input energy required by the designed filters (a). Magnitude FRF of the optimal filters (PM, ACM, EDM) for loudspeaker \#5 normalized by the response of the DAS filter for loudspeaker \#5 (b). The vertical dashed lines are the frequencies indicating the three regions for the front beam. . . . . . . . . 25

8 Front beam. Calculated regularization parameters for PM and ACM method (a) and $\alpha$ for the EDM method

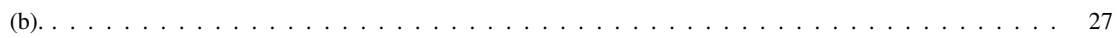

9 Front beam. FRFs of the compensation filters $W / W_{\text {DAS }}$ for the optimal methods. Magnitude (a) and phase

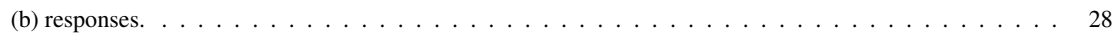

10 Front beam. Impulse responses of the filters for loudspeaker \#5 for the PM (a) and EDM (b) methods. The FRFs of both filters is shown in Fig. 7 (b) . . . . . . . . . . . . . . . . . . . . . . . . . 29

11 Simulated (a) and measured (b) array efficiency for the front beam due to filters designed with four beam-

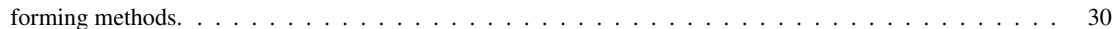

12 Simulated (a) and measured (b) acoustic contrast for the front beam due to filters designed with four beam-

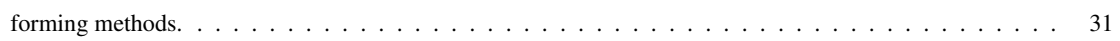

13 Front Beam. FRFs (a) and IRs (b) of the measured filters responses of the DAS and PM methods and $\tilde{H}$. . . 33

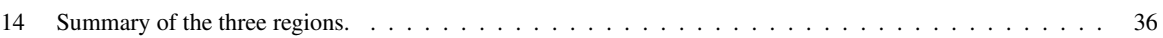

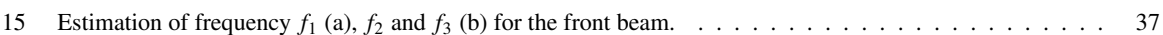

16 Side beam. Input energy required by the designed filters (a). Magnitude FRF of the optimal filters (PM, ACM, EDM) for loudspeaker \#5 normalized by the response of the DAS filter for loudspeaker \#5 (b). The vertical dashed lines are the frequencies indicating the three regions for the side beam. . . . . . . . . 38

17 Simulated (a) and measured (b) array efficiency for the side beam due to filters designed with four beamforming methods. . . . . . . . . . . . . . . . . . . . . . . 39

18 Simulated (a) and measured (b) acoustic contrast for the side beam due to filters designed with four beam-

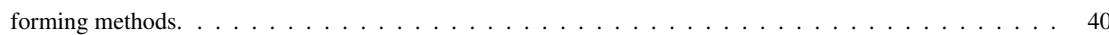


19 Procedure for the calculation of $\alpha_{\mathrm{MAX}}$. Acoustic contrast as a function of $\alpha$ (a). Second partial derivative of the acoustic contrast with respect to $\alpha$ at $125 \mathrm{~Hz}$ (b) and $2 \mathrm{kHz}$ (c), respectively, and selected values of $\alpha_{\mathrm{MAX}}$ (indicated by the arrows). Selected $\alpha_{\mathrm{MAX}}$ as a function of frequency (d) using the procedure described in

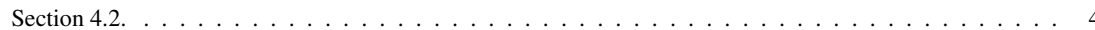

